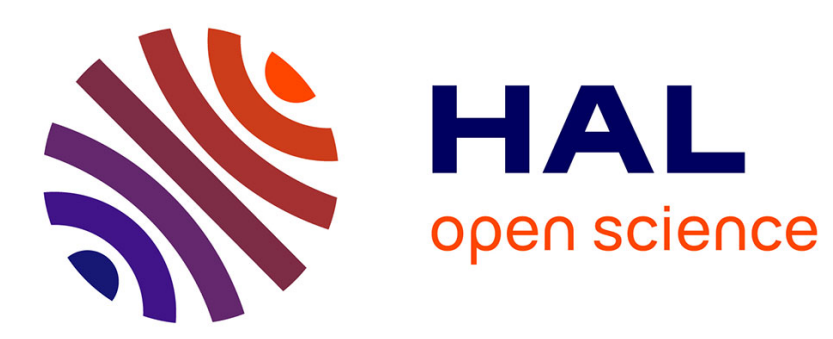

\title{
Numerical implementation of static Field Dislocation Mechanics theory for periodic media
}

Renald Brenner, Armand Jr. Beaudoin, Pierre Suquet, Amit Acharya

\section{To cite this version:}

Renald Brenner, Armand Jr. Beaudoin, Pierre Suquet, Amit Acharya. Numerical implementation of static Field Dislocation Mechanics theory for periodic media. 2013. hal-00918607

\section{HAL Id: hal-00918607 https://hal.science/hal-00918607}

Preprint submitted on 16 Dec 2013

HAL is a multi-disciplinary open access archive for the deposit and dissemination of scientific research documents, whether they are published or not. The documents may come from teaching and research institutions in France or abroad, or from public or private research centers.
L'archive ouverte pluridisciplinaire HAL, est destinée au dépôt et à la diffusion de documents scientifiques de niveau recherche, publiés ou non, émanant des établissements d'enseignement et de recherche français ou étrangers, des laboratoires publics ou privés. 


\title{
Numerical implementation of static Field Dislocation Mechanics theory for periodic media
}

\author{
R. Brenner ${ }^{a *}$, A. J. Beaudoin ${ }^{\mathrm{b}}$, P. Suquet ${ }^{\mathrm{c}}$ and A. Acharya ${ }^{\mathrm{d}}$ \\ ${ }^{a}$ Institut Jean Le Rond d'Alembert, Université Pierre et Marie Curie, CNRS, UMR 7190, \\ 4 place Jussieu, 75252 Paris Cedex 05, France; ${ }^{b}$ Department of Mechanical Science and \\ Engineering, University of Illinois at Urbana-Champaign, 1206 W. Green St., Urbana, \\ IL61801, USA; ${ }^{\mathrm{c}}$ Laboratoire de Mécanique et d'Acoustique, CNRS, UPR 7051, Aix \\ Marseille Univ., Centrale Marseille, 31 chemin Joseph Aiguier, 13402 Marseille Cedex \\ 20, France; ${ }^{\mathrm{d}}$ Department of Civil and Environmental Engineering, Carnegie Mellon \\ University, 103 Porter Hall, Pittsburgh, PA 15213, USA;
}

\begin{abstract}
This paper investigates the implementation of Field Dislocation Mechanics theory for media with a periodic microstructure (i.e. the Nye dislocation tensor and the elastic moduli tensor are considered as spatially periodic continuous fields). In this context, the uniqueness of the stress and elastic distortion fields is established. This allows to propose an efficient numerical scheme based on Fourier transform to compute the internal stress field, for a given spatial distribution of dislocations and applied macroscopic stress. This numerical implementation is assessed by comparison with analytical solutions for homogeneous as well as heterogeneous elastic media. A particular insight is given to the critical case of stress-free dislocation microstructures which represent equilibrium solutions of the Field Dislocation Mechanics theory.
\end{abstract}

Keywords: Field Dislocation Mechanics; internal stresses; periodic microstructure; Fourier transform; infinite bicrystal; zero-stress dislocation distributions

\section{Introduction}

The Field Dislocation Mechanics (FDM) theory developed by Acharya [1, 2] relates the Elastic theory of Continuously Distributed Dislocations (ECDD) [3] to models of mesoscale elastoplasticity. The finite-element implementation of this physicallybased approach has allowed to study important features of plasticity such as size effects and the development of back-stress, among others $[4,5]$. In the absence of evolution of the Nye's dislocation density tensor, the stress field solution of the equations of the FDM theory is exactly the one of the ECDD [1]. This static problem is a key prerequisite to the plastic evolution problem.

An efficient numerical scheme based on fast Fourier transform (FFT) has been proposed to solve periodic boundary-value (PBV) problems in continuum mechanics [6]. This numerical approach solves the integral equation of the PBV problem by means of the Green's function of a reference medium. It offers an attractive alternative to the finite-element method for periodic problems and has been successfully applied, up to now, in the context of elastic and elastoplastic composites and polycrystals $[6-10]$.

The numerical implementation of the FDM theory by means of the FFT framework would allow consideration, with reasonable computational capacities, of mi-

*Corresponding author. Email: renald.brenner@upmc.fr 
crostructurally complex problems (3D polycrystalline aggregates, for instance). However, its feasibility has not yet been explored in details. The present article reports preliminary investigations towards this goal. Specifically, the aim of this work is to investigate first the static FDM problem in a linear periodic elastic framework. In Section 2, the governing equations of static FDM are recalled and the uniqueness of the fields solving the local problem is investigated. Then, making use of a Stokes-Helmholtz ( $\mathrm{SH}$ ) like decomposition of the elastic distorsion, the expression of the stress field solution of the anisotropic (homogeneous or heterogeneous) elastic problem is derived. Section 3 reports on some FFT computations of the internal stress field, for a fixed dislocation density tensor field, which are compared with available analytical solutions. In particular, attention is paid to stress-free dislocation microstructures which are equilibrium solutions of the FDM theory [4].

\section{Static Field Dislocation Mechanics theory}

\subsection{Governing equations}

The problem we are addressing is the determination of the internal stress field generated by a given dislocation density field [1,3], and an applied macroscopic stress, in a heterogeneous anisotropic elastic media. The Nye dislocation tensor $\boldsymbol{\alpha}$ [11] and the elastic moduli tensor $\mathbf{C}$ are considered as periodic continuous fields. The problem at hand reads as follows. Given a periodic dislocation field $\boldsymbol{\alpha}$ and a macroscopic stress $\overline{\boldsymbol{\sigma}}$, find the elastic distortion $\mathbf{U}^{e}$ and stress $\boldsymbol{\sigma}$ fields which solve:

$$
\left\{\begin{array}{l}
\operatorname{curl}\left(\mathbf{U}^{\mathrm{e}}\right)=\boldsymbol{\alpha}, \quad \operatorname{div}(\boldsymbol{\sigma})=\mathbf{0}, \quad \boldsymbol{\sigma}=\mathbf{C}: \mathbf{U}^{\mathrm{e}} \quad \text { in } \mathbb{R}^{N}, \\
\mathrm{U}^{\mathrm{e}} \text { periodic, } \quad \boldsymbol{\sigma} \text { periodic, } \quad\langle\boldsymbol{\sigma}\rangle=\overline{\boldsymbol{\sigma}}
\end{array}\right.
$$

where $\langle$.$\rangle denotes the spatial average over the unit-cell V$. From the definition of the Nye tensor, the classical relation follows

$$
\operatorname{div}(\boldsymbol{\alpha})=\mathbf{0} \quad \text { in } \mathbb{R}^{N}
$$

whose physical meaning is that dislocations cannot end within the material (they either form loops or reach the surface). Besides, since the average on the unit-cell of the gradient of a periodic function is zero, the periodicity of $\mathbf{U}^{e}$ implies that

$$
\left\langle\operatorname{curl}\left(\mathbf{U}^{\mathrm{e}}\right)\right\rangle=\langle\boldsymbol{\alpha}\rangle=\mathbf{0}
$$

In other words, a periodic $\mathbf{U}^{\mathrm{e}}$ field cannot be found if $\boldsymbol{\alpha}$ is constant ${ }^{1}$.

Proposition: Assume that $\boldsymbol{\alpha}$ is a second-order tensor field such that

$$
\boldsymbol{\alpha} \in \mathcal{L}_{\sharp}^{2}(V), \quad \operatorname{div}(\boldsymbol{\alpha})=\mathbf{0} \text { in } \mathbb{R}^{N}, \quad\langle\boldsymbol{\alpha}\rangle=\mathbf{0},
$$

\footnotetext{
${ }^{1}$ This can be shown by prescribing $\boldsymbol{\alpha}=c \mathbf{e}_{1} \otimes \mathbf{e}_{3}$. In this case, we have

$$
U_{12,1}^{\mathrm{e}}-U_{11,2}^{\mathrm{e}}=c
$$

which admits non-periodic solutions, for instance,

$$
\mathbf{U}^{\mathrm{e}}=\frac{c\left(x_{1}+x_{2}\right)}{2}\left(\mathbf{e}_{1} \otimes \mathbf{e}_{2}-\mathbf{e}_{1} \otimes \mathbf{e}_{1}\right) .
$$
}


and that the heterogeneous stiffness tensor $\mathbf{C}(\mathbf{x})$ has the usual minor and major symmetries, is uniformly bounded and coercive on $V$. Then the problem (1) admits solution fields $\left(\boldsymbol{\sigma}, \mathbf{U}^{e}\right)$ in $\mathcal{L}_{\sharp}^{2}(V) \times \mathcal{L}_{\sharp}^{2}(V)$ with $\boldsymbol{\sigma}$ being unique and $\mathbf{U}^{e}$ being unique up to a constant skew-symmetric tensor.

Proof $^{1}$ : Making use of lemma B.2 (Appendix B), select a $\chi$ field satisfying (B7) and look for a periodic potential field $z$ such that

$$
\left\{\begin{array}{l}
\boldsymbol{\sigma}=\mathbf{C}:(\boldsymbol{\nabla} z+\boldsymbol{\chi}), \quad \operatorname{div}(\boldsymbol{\sigma})=\mathbf{0} \quad \text { in } \mathbb{R}^{N}, \\
z \in \boldsymbol{H}_{\sharp}^{1}(V), \quad \boldsymbol{\sigma} \in \mathcal{L}_{\sharp}^{2}(V), \quad\langle\boldsymbol{\sigma}\rangle=\overline{\boldsymbol{\sigma}} .
\end{array}\right.
$$

(5) is a periodic thermoelastic problem where $-\chi$ plays the role of a known thermal strain. By classical arguments, it admits solution fields $(\boldsymbol{z}, \boldsymbol{\sigma})$. The solution $\mathbf{U}^{\mathrm{e}}$ to (1) is $\mathbf{U}^{\mathrm{e}}=\boldsymbol{\nabla} z+\chi$. This completes the proof of the existence of a solution.

Assume that the problem (1) admits two solutions $\left(\boldsymbol{\sigma}_{1}, \mathbf{U}_{1}^{\mathrm{e}}\right)$ and $\left(\boldsymbol{\sigma}_{2}, \mathbf{U}_{2}^{\mathrm{e}}\right)$ and set

$$
\boldsymbol{\sigma}_{*}=\sigma_{1}-\boldsymbol{\sigma}_{2}, \quad \mathbf{U}_{*}^{\mathrm{e}}=\mathbf{U}_{1}^{\mathrm{e}}-\mathbf{U}_{2}^{\mathrm{e}} .
$$

Then the fields $\boldsymbol{\sigma}_{*}$ and $\mathbf{U}_{*}^{\mathrm{e}}$ solve

$$
\left\{\begin{array}{l}
\operatorname{curl}\left(\mathbf{U}_{*}^{\mathrm{e}}\right)=\mathbf{0}, \quad \operatorname{div}\left(\boldsymbol{\sigma}_{*}\right)=\mathbf{0}, \quad \boldsymbol{\sigma}_{*}=\mathbf{C}: \mathbf{U}_{*}^{\mathrm{e}} \quad \text { in } \mathbb{R}^{N}, \\
\mathbf{U}_{*}^{\mathrm{e}} \in \mathcal{L}_{\sharp}^{2}(V), \quad \boldsymbol{\sigma}_{*} \in \mathcal{L}_{\sharp}^{2}(V), \quad\left\langle\boldsymbol{\sigma}_{*}\right\rangle=\mathbf{0} .
\end{array}\right.
$$

From lemma B.1 (Appendix B), there exists a periodic field $\boldsymbol{z}_{*}$ in $\boldsymbol{H}_{\sharp}^{1}(V)$ such that

$$
\mathbf{U}_{*}^{\mathrm{e}}=\nabla z_{*}+\left\langle\mathbf{U}_{*}^{\mathrm{e}}\right\rangle .
$$

Then

$$
\left\langle\boldsymbol{\sigma}_{*}: \mathbf{U}_{*}^{\mathrm{e}}\right\rangle=\left\langle\boldsymbol{\sigma}_{*}: \nabla z_{*}\right\rangle+\left\langle\boldsymbol{\sigma}_{*}\right\rangle:\left\langle\mathbf{U}_{*}^{\mathrm{e}}\right\rangle,
$$

but since $\left\langle\boldsymbol{\sigma}_{*}\right\rangle=\mathbf{0}$, the only term contributing in the right-hand-side of (9) is $\left\langle\sigma_{*}: \nabla z_{*}\right\rangle$. Note that

$$
\left\langle\boldsymbol{\sigma}_{*}: \nabla z_{*}\right\rangle=-\left\langle\operatorname{div}\left(\boldsymbol{\sigma}_{*}\right) \cdot z_{*}\right\rangle+\frac{1}{|V|} \int_{\partial V} \boldsymbol{\sigma}_{*} \cdot \mathbf{n} . z_{*} \mathrm{~d} s .
$$

$\boldsymbol{\sigma}_{*}$ is a divergence-free field, so that the first term in the right-hand-side of (10) vanishes. Finally, $\boldsymbol{\sigma}_{*} \cdot \mathbf{n}$ takes opposite values on opposite sides of $V$ whereas $\boldsymbol{z}_{*}$ take identical values. Therefore, the integral on the boundary of the unit-cell $\partial V$ is zero. Coming back to (9), it is found that

$$
\left\langle\mathbf{U}_{*}^{\mathrm{e}}: \mathbf{C}: \mathbf{U}_{*}^{\mathrm{e}}\right\rangle=\left\langle\boldsymbol{\sigma}_{*}: \mathbf{U}_{*}^{\mathrm{e}}\right\rangle=0
$$

$\mathbf{U}_{*}^{\mathrm{e}}$ is thus a pointwise skew-symmetric field. Due to the minor symmetry of $\mathbf{C}$, $\boldsymbol{\sigma}_{*}=\mathbf{0}$. This completes the proof of the uniqueness of the stress solution field.

In addition, since $\mathbf{U}_{*}^{\mathrm{e}}$ is a curl-free and skew-symmetric field, it follows from the decomposition (8) that $\varepsilon\left(z_{*}\right)=\mathbf{0}$, where $\boldsymbol{\varepsilon}$ denotes the symmetric part of the

${ }^{1}$ The functional spaces $\mathcal{L}_{\sharp}^{2}(V)$ and $\boldsymbol{H}_{\sharp}^{1}(V)$ are defined in Appendix B. 
gradient. According to Temam [12], the kernel of $\varepsilon$ in the distributional sense on $\mathbb{R}^{N}$ is composed of rigid-body displacements and therefore $z_{*}=\mathbf{a}+\boldsymbol{\Omega}$. $\mathbf{x}$ where $\boldsymbol{\Omega}$ is a skew-symmetric tensor. Besides, the periodicity of $\boldsymbol{z}_{*}$ implies that $\boldsymbol{\Omega}=\mathbf{0}$ since $\left\langle\boldsymbol{\nabla} \boldsymbol{z}_{*}\right\rangle=\mathbf{0}$. It follows from (8) that $\mathbf{U}_{*}^{\mathrm{e}}$ is a uniform skew-symmetric field.

\subsection{Fourier transform-based numerical scheme}

Using a SH-like decomposition of the elastic distortion

$$
\mathbf{U}^{\mathrm{e}}=\nabla z+\chi
$$

the problem (1) can be alternatively written

$$
\begin{cases}\operatorname{curl}(\chi)=\boldsymbol{\alpha}, & \operatorname{div}(\chi)=\mathbf{0} \quad \text { in } \mathbb{R}^{N}, \\ \operatorname{div}(\boldsymbol{\sigma})=\mathbf{0}, & \boldsymbol{\sigma}=\mathbf{C}:(\boldsymbol{\nabla} z+\chi) \quad \text { in } \mathbb{R}^{N}, \\ \chi \in \mathcal{H}_{\sharp}^{1}(V), & \boldsymbol{z} \in \boldsymbol{H}_{\sharp}^{1}(V), \quad \boldsymbol{\sigma} \in \mathcal{L}_{\sharp}^{2}(V), \\ \langle\boldsymbol{\sigma}\rangle=\overline{\boldsymbol{\sigma}} . & \end{cases}
$$

The periodic local problem, as laid out above, can be efficiently solved by using the standard Green's function method and the Fourier transform technique. We detail its resolution by distinguishing two situations: elastically homogeneous or heterogeneous material.

\subsubsection{Homogeneous elasticity}

In the case of a homogeneous elastic medium with moduli tensor $\mathbf{C}(\mathbf{x}) \equiv \mathbf{C}^{0}$, the solution field $\varepsilon$ (symmetric part of $\boldsymbol{\nabla} \boldsymbol{z}$ ) of the local problem (13) can be classically expressed as

$$
\varepsilon(\mathbf{x})=\langle\varepsilon\rangle-\left(\Gamma^{0} * \boldsymbol{\tau}\right)(\mathbf{x}) \quad \text { where } \quad \boldsymbol{\tau}=\mathbf{C}^{0}: \chi
$$

* is the convolution product and $\Gamma^{0}$ is the Green operator of the homogeneous medium with elasticity $\mathbf{C}^{0}$. The Fourier transform $\widehat{\varepsilon}$ reads

$$
\widehat{\boldsymbol{\varepsilon}}(\boldsymbol{\xi})=-\widehat{\boldsymbol{\Gamma}}^{0}(\boldsymbol{\xi}): \widehat{\boldsymbol{\tau}}(\boldsymbol{\xi}), \quad \forall \boldsymbol{\xi} \neq 0
$$

and the elastic stress field, in the Fourier space, is obtained by the constitutive relation as

$$
\widehat{\boldsymbol{\sigma}}(\boldsymbol{\xi})=\left(\mathbf{I}-\mathbf{C}^{0}: \widehat{\boldsymbol{\Gamma}}^{0}(\boldsymbol{\xi})\right): \mathbf{C}^{0}: \widehat{\chi}(\boldsymbol{\xi}), \quad \forall \boldsymbol{\xi} \neq 0, \quad \widehat{\boldsymbol{\sigma}}(\mathbf{0})=\overline{\boldsymbol{\sigma}}
$$

with $\mathbf{I}$ the fourth-order identity tensor. By construction (Appendix B), the Fourier transform of the $\chi$ field reads

$$
\widehat{\chi}(\boldsymbol{\xi})=-\imath \frac{\widehat{\boldsymbol{\alpha}}(\boldsymbol{\xi}) \times \boldsymbol{\xi}}{\|\boldsymbol{\xi}\|^{2}}, \forall \boldsymbol{\xi} \neq \mathbf{0}, \quad \widehat{\chi}(\mathbf{0})=\mathbf{0}
$$

with $\imath$ the imaginary unit, and the expression of the Fourier transform of the Green operator is

$$
\widehat{\boldsymbol{\Gamma}}^{0}(\boldsymbol{\xi})=\left[\boldsymbol{\xi} \otimes\left(\boldsymbol{\xi} \cdot \mathbf{C}^{0} \cdot \boldsymbol{\xi}\right)^{-1} \otimes \boldsymbol{\xi}\right]^{(s)}
$$


where $[.]^{(s)}$ indicates minor and major symmetrization. Note that the constraint (17) on the average of $\boldsymbol{\chi}$ implies that the average eigenstress $\langle\boldsymbol{\tau}\rangle$ is zero since the medium is elastically homogeneous. It follows that $\langle\varepsilon\rangle=\mathbf{C}^{0^{-1}}: \overline{\boldsymbol{\sigma}}$. Finally, it is worth mentioning that if the condition $\langle\boldsymbol{\alpha}\rangle=\mathbf{0}$ is not met, the correct stress field is nevertheless obtained with (16) since the stress field due to a constant dislocation density (i.e. $\widehat{\boldsymbol{\alpha}}(\boldsymbol{\xi})=\mathbf{0}, \forall \boldsymbol{\xi} \neq \mathbf{0}$ ) is identically null.

\subsubsection{Heterogeneous elasticity}

To address the case of a heterogeneous elastic medium, a common practice in micromechanics is to introduce a reference medium with homogeneous elasticity $\mathbf{C}^{0}$. The local problem (13) is thus transformed into

$$
\begin{cases}\operatorname{curl}(\chi)=\boldsymbol{\alpha}, & \operatorname{div}(\chi)=\mathbf{0} \quad \text { in } \mathbb{R}^{N}, \\ \operatorname{div}(\boldsymbol{\sigma})=\mathbf{0}, & \boldsymbol{\sigma}=\mathbf{C}^{0}: \boldsymbol{\nabla} z+\boldsymbol{\tau} \text { in } \mathbb{R}^{N}, \\ \chi \in \mathcal{H}_{\sharp}^{1}(V), & \boldsymbol{z} \in \boldsymbol{H}_{\sharp}^{1}(V), \quad \boldsymbol{\sigma} \in \mathcal{L}_{\sharp}^{2}(V), \\ \langle\boldsymbol{\sigma}\rangle=\overline{\boldsymbol{\sigma}}, & \end{cases}
$$

where

$$
\boldsymbol{\tau}=\mathbf{C}: \chi+\left(\mathbf{C}-\mathbf{C}^{0}\right): \nabla z
$$

The only difference with the previous homogeneous elastic problem is that the prescribed eigenstress field $\boldsymbol{\tau}$ is not known a priori since it depends on the field $z$ which solves the problem. When the reference medium is adequately chosen, the solution field $\varepsilon$ is obtained as a series expansion reading [13]

$$
\varepsilon(\mathbf{x})=\sum_{i=0}^{+\infty}\left(-\boldsymbol{\Gamma}^{0} * \delta \mathbf{C}(\mathbf{x})\right)^{i}:\left(\langle\varepsilon\rangle-\left(\boldsymbol{\Gamma}^{0} * \mathbf{C}: \boldsymbol{\chi}\right)(\mathbf{x})\right)
$$

Based on this expansion, an efficient iterative numerical procedure using fastFourier transforms (FFT) has been proposed to solve (19) [6]. The reader is also referred to [14] for a comprehensive comparative study of numerical schemes based on other iterative methods.

\section{Assessment of stress field computations}

We present illustrative examples comparing the internal elastic stress field obtained by the FFT numerical scheme with analytical solutions.

\subsection{Screw dislocation}

The stress field created by a single screw dislocation in an infinite isotropic medium without applied macroscopic stress can be envisaged in a periodic framework with a sufficiently large unit-cell. In this case, we have $\langle\boldsymbol{\alpha}\rangle \neq \mathbf{0}$ and the non-periodic part of the $\mathbf{U}^{\mathrm{e}}$ field cannot be obtained in this setting. By contrast, the stress field solution can be investigated since a constant dislocation density over the unit-cell results in a null stress field. However, attention has to be paid to the modelling of the single dislocation to get an accurate solution. The analytical expression of the shear stresses created by a single screw dislocation, placed at the origin of the 
reference frame, reads [15]

$$
\sigma_{13}\left(x_{1}, x_{2}\right)=-\frac{\mu b}{2 \pi} \frac{x_{2}}{\left(x_{1}^{2}+x_{2}^{2}\right)} \quad \text { and } \quad \sigma_{23}\left(x_{1}, x_{2}\right)=\frac{\mu b}{2 \pi} \frac{x_{1}}{\left(x_{1}^{2}+x_{2}^{2}\right)} .
$$

By definition, the Burgers vector $\mathbf{b}$ for a closed circuit limiting a surface $S$ and the dislocation tensor $\boldsymbol{\alpha}$ are related by

$$
\mathbf{b}=\int_{S} \boldsymbol{\alpha}(\mathbf{x}) \cdot \mathbf{n} \mathrm{d} s
$$

with $\mathbf{n}$ the unit normal to the surface $S$. To model a straight screw dislocation along the $\mathbf{x}_{3}$ axis, a density $\alpha_{33}$ has to be prescribed. The Burgers vector, for a surface $S$ normal to $\mathbf{x}_{3}$-axis, then reads

$$
\mathbf{b}=b \mathbf{e}_{3}=\int_{S} \alpha_{33}(\mathbf{x}) \mathrm{d} s \mathbf{e}_{3}
$$

In this configuration, the dislocation density is thus zero in the whole unit-cell except at the dislocation location. In the context of Fourier transforms, this discontinuity results in stress oscillations due to the Gibbs phenomenon. To reduce this undesirable effect, we have considered different distributions of the dislocation density $\alpha_{33}$. This can be viewed as a "numerical spreading" of the core of the dislocation. Even if no core dislocation energy is accounted for in the present setting, this spreading of the dislocation is physically realistic. We have considered a unit-cell domain of $318 b \times 318 b$, with $b$ the norm of the Burgers vector, and the screw dislocation was placed at the center of the cell discretized on a regular grid of $1024 \times 1024$ pixels. Material data corresponding to aluminium are considered, namely : $b=0.286 \mathrm{~nm}, \mu=23 \mathrm{GPa}$ and $\nu=0.36$. The results obtained with three dislocation distributions to model the straight screw dislocation are reported in Figure 1. If the dislocation density is prescribed on a single pixel, strong stress oscillations arise, as expected. These oscillations can be strongly attenuated if the dislocation density is uniformly prescribed on a surface of $3 \times 3$ pixels. For the present calculation, it corresponds to a spreading of the dislocation of extent $\sim b$. Further, our results show that a smoother triangular distribution over the same surface area leads to a stress field with no oscillations which coincides with the analytical solution for an infinite medium.

\subsection{Edge-dislocation dipole}

We investigate the stress field created by an edge dislocation dipole in an infinite homogeneous elastic medium without applied macroscopic stress. To do so, we consider a 2D unit-cell which is large compared to the dipole spacing and we prescribe $\overline{\boldsymbol{\sigma}}=\mathbf{0}$. The unit-cell domain and the material data are the same as for the case of the screw dislocation (Subsection 3.1). The dislocation dipole was prescribed by placing positive and negative dislocation densities at $\mathbf{x}^{A}=(10 b, 10 b)$ and $\mathbf{x}^{B}=(-10 b,-10 b)$, respectively. In practice, the dislocation density $\alpha$ has been allocated, for each dislocation, on a surface area of $3 \times 3$ pixels to avoid the Gibbs phenomenon when computing Fourier transforms (see Subsection 3.1). The size of this spread is of the order of the Burgers vector.

The $\sigma_{11}$ component of the stress field created by a single edge dislocation, placed 


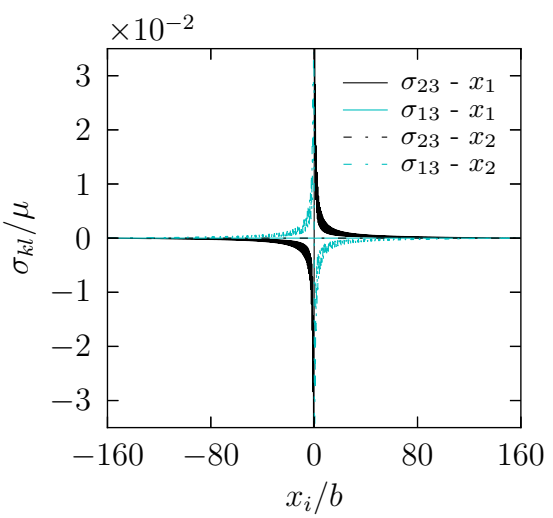

(a)

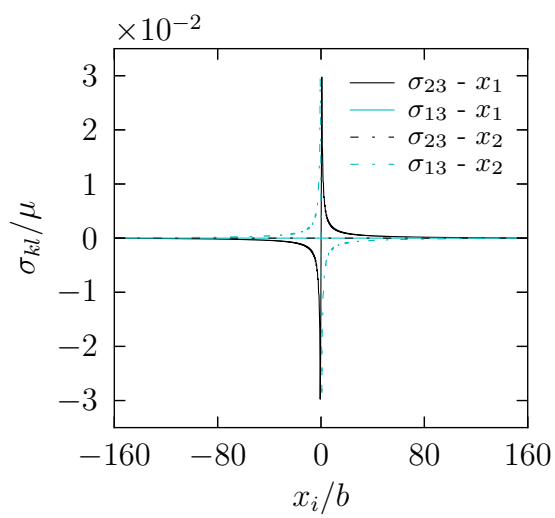

(b)

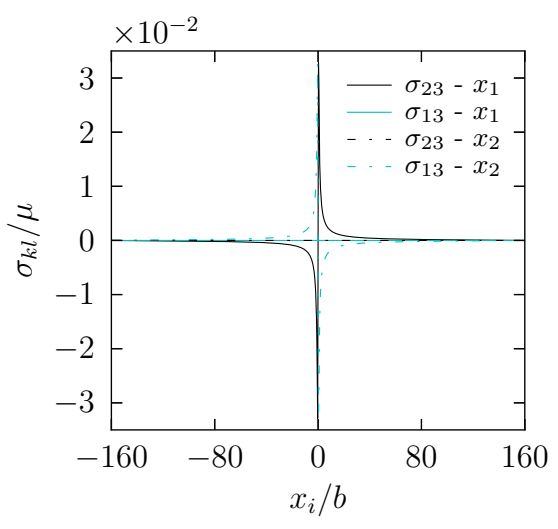

(c)

Figure 1. Numerical stress field of a single screw dislocation obtained with the FFT scheme for different distributions of the dislocation density. (a) single pixel, (b) uniform and (c) "hut" shape distributions over a surface $S=3 \times 3$ pixels.

at the origin of the reference frame, reads [15]

$$
\sigma_{11}\left(x_{1}, x_{2}\right)=-\frac{\mu b}{2 \pi(1-\nu)} \frac{x_{2}\left(3 x_{1}^{2}+x_{2}^{2}\right)}{\left(x_{1}^{2}+x_{2}^{2}\right)^{2}}
$$

The analytical stress field of the dipole is obtained with the superposition principle. Comparisons between the FFT and analytical stress fields are reported in Figure 2. The 2D plot highlights the correct description of the constant stress contours about each dislocation. Besides, the quantitative comparison of the stress field shows a perfect agreement of the FFT numerical result.

\subsection{Griffith-Inglis crack}

The stress field in an infinite homogeneous medium containing a crack in mode I is considered. This problem is known as the Griffith-Inglis crack [16, 17]. In the present effort, we adopt the dislocation-based description of cracks [18, 19]. A similar problem has been addressed independently in [20]. The crack is modelled by a continuous distribution of dislocations such that the faces of the crack are traction free (i.e. the stress field generated by the dislocation distribution must balance the applied stress along the crack). For a mode I crack lying in the $\left(\mathbf{x}_{1}, \mathbf{x}_{3}\right)$ plane, with crack tips at $x_{1}= \pm a$ and infinitely long in the $\mathbf{x}_{3}$ direction, the following density 


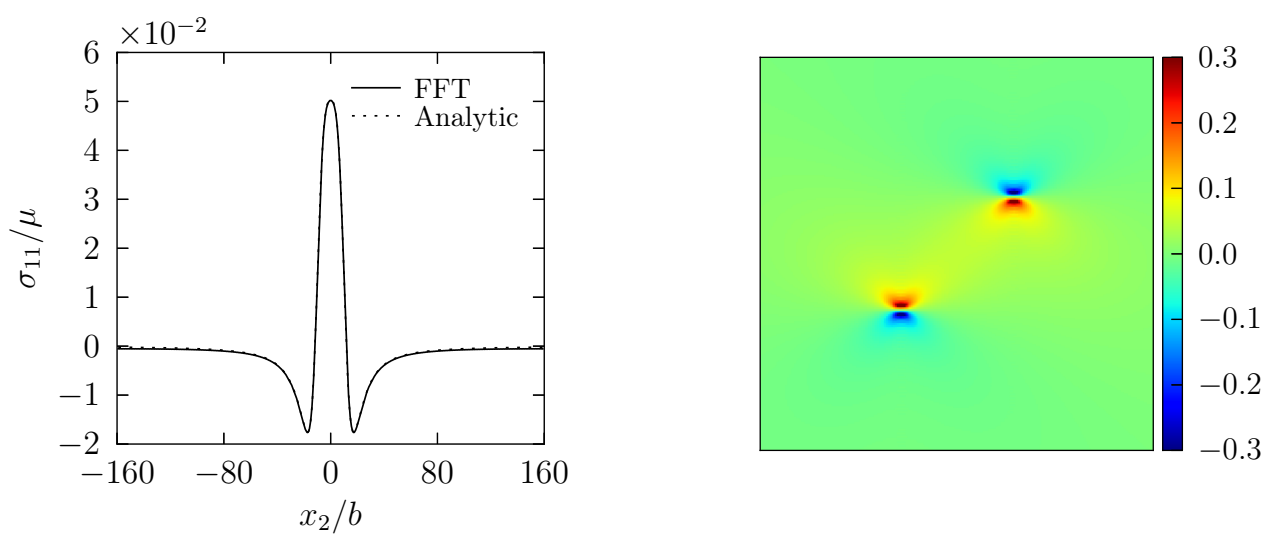

Figure 2. Component $\sigma_{11}$ of the stress field of an edge dislocation dipole. Left: Fluctuation along the $\mathbf{x}_{2}$ axis at $x_{1}=0$. Right: FFT result of the $2 \mathrm{D} \sigma_{11}$ field (zoom). The results are normalized by the shear modulus $\mu$.

of climb edge dislocations (i.e. edge dislocations with Burgers vector normal to the crack plane) has to be prescribed [18]

$$
\alpha_{23}\left(x_{1}, x_{2}\right)=\frac{\sigma_{\infty}}{w\left(x_{2}\right)} \frac{2(1-\nu)}{\mu} \frac{x_{1}}{\sqrt{a^{2}-x_{1}^{2}}}, \quad \forall x_{1} \in[-a ; a]
$$

with $\sigma_{\infty}$ the applied tensile stress normal to the crack plane. The function $w\left(x_{2}\right)$ represents a weighting that is applied across the faces of the crack, with again the purpose of "spreading the core" perpendicular to the crack plane. Specifically,

$$
\int_{-d}^{d} w\left(x_{2}\right) \mathrm{d} x_{2}=\delta x_{2}
$$

The Burgers vector content between $x_{1}$ and $x_{1}+\delta x_{1}$ is $\alpha_{23}\left(x_{1}, x_{2}\right) \delta x_{1} \delta x_{2}$. A simple triangular distribution across five cells (i.e. approximately $1.5 b$ ) was found to be adequate for the present computation. Another consideration in prescribing the dislocation density lies in the singularity of (26) at the crack tips. This was handled by evaluating $\alpha_{23}\left(x_{1}, x_{2}\right)$ at the edge of the cell, for cells lying at $x_{1}=$ $\pm a$, and assigning that value to the center of the cell. The numerical stress field calculation has been performed for a crack of width $2 a=34.8 b$ in a unit-cell domain of $318 b \times 318 b$. The prescribed average stress is a uniaxial tension normal to the crack plane $\left(\overline{\boldsymbol{\sigma}}=\sigma_{\infty} \mathbf{e}_{2} \otimes \mathbf{e}_{2}\right)$. The FFT numerical result has been quantitatively compared with the analytical expression of the tensile stress in the crack plane

$$
\sigma_{22}\left(x_{1}, x_{2}=0\right)= \begin{cases}\sigma_{\infty} \frac{\left|x_{1}\right|}{\sqrt{x_{1}^{2}-a^{2}}} & \text { if }\left|x_{1}\right| \geq a \\ 0 & \text { otherwise }\end{cases}
$$

A very good match is obtained for the tensile stress prediction along the crack plane and the 2D isostress contours are correctly described [21] (Figure 3). 

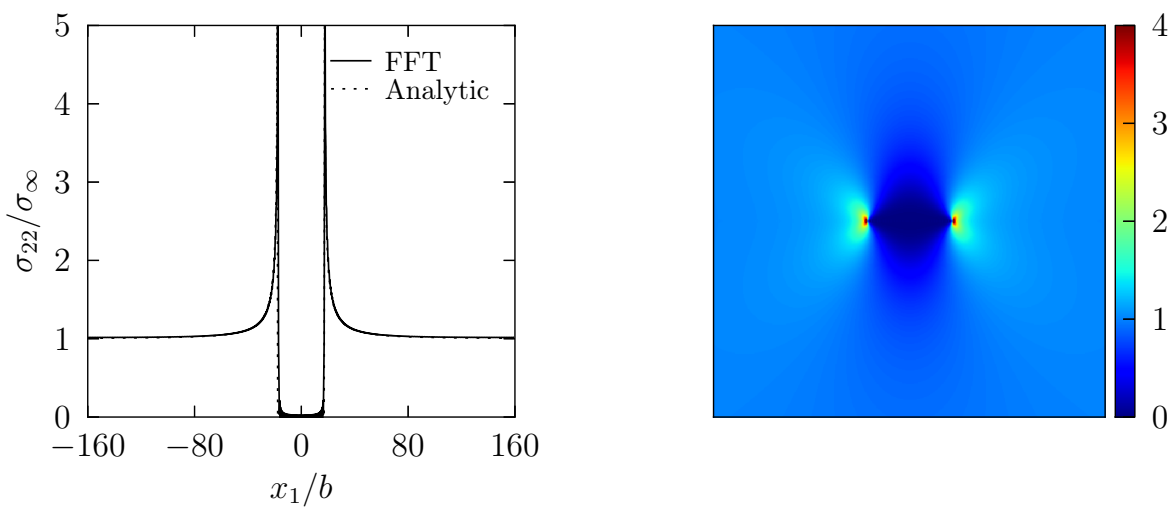

Figure 3. Tensile stress component for a Griffith-Inglis crack. Left: Variation in the plane of the crack. Right: FFT result of the 2D field. The results are normalized by the applied tensile stress $\sigma_{\infty}$

\subsection{Stress-free dislocation distributions}

Equilibrated dislocations distributions with a pointwise zero-stress field exist in materials and provide interesting and critical cases for a further assessment of the accuracy of FFT computations. Such configurations are equally called impotent or zero-stress everywhere (ZSE) dislocation distributions [22, 23].

The general condition for a stress-free dislocation field $\boldsymbol{\alpha}$ can be obtained as follows. Since the elastic tensor $\mathbf{C}$ has minor symmetry, a stress-free state implies that the elastic distorsion $\mathbf{U}^{e}$ is skew-symmetric, that is

$$
\mathbf{U}^{e}=\boldsymbol{\epsilon} \cdot \varphi
$$

with $\varphi$ an arbitrary vector field. From the definitions of the Nye tensor and the elastic distorsion, we have

$$
\boldsymbol{\alpha}=\operatorname{curl}\left(\mathbf{U}^{e}\right)=\nabla \times(\boldsymbol{\epsilon} \cdot \boldsymbol{\varphi})={ }^{\mathrm{T}} \nabla \varphi-\operatorname{tr}(\boldsymbol{\nabla} \varphi) \mathbf{i} .
$$

with $\mathbf{i}$ the second-order identity tensor. The stress-free dislocation field $\boldsymbol{\alpha}$ is thus a function of the gradient of the (arbitrary) vector field $\varphi$. In a cartesian coordinate system, its components read

$$
\alpha_{i m}=\epsilon_{m j k} \epsilon_{i k r} \varphi_{r, j}=\varphi_{m, i}-\varphi_{k, k} \delta_{i m} .
$$

This method for building a stress-free dislocation field has been used for finiteelement computations in a non-periodic context [4]. Obviously, the expression of the stress-free dislocation field (30) is independent of the elastic tensor field. It is thus valid in the general case of a heterogeneous anisotropic elastic medium. This is in contrast with other investigations on stress-free dislocation fields assuming homogeneous and isotropic elasticity [24]. By adopting the decomposition (12) of the elastic distorsion, we can first determine the $\chi$ field which solves

$$
\operatorname{curl}(\chi)=\alpha, \quad \operatorname{div}(\chi)=0, \quad\langle\chi\rangle=0, \quad \chi \in \mathcal{H}_{\sharp}^{1}(V),
$$

and then find the potential field $z$, whose gradient (i.e. compatible part of $\mathbf{U}^{\mathrm{e}}$ ) counterbalances the symmetric part of $\boldsymbol{\chi}$, as a solution of

$$
\operatorname{div}(\boldsymbol{\sigma})=\mathbf{0}, \quad \boldsymbol{\sigma}=\mathbf{C}:(\boldsymbol{\nabla} z+\chi), \quad \boldsymbol{\sigma}=\mathbf{0}, \quad z \in \boldsymbol{H}_{\sharp}^{1}(V), \quad \boldsymbol{\sigma} \in \mathcal{L}_{\sharp}^{2}(V),
$$


which admits the unique elastic distortion $\mathbf{U}^{\mathrm{e}}$ and stress solution fields with $\boldsymbol{\sigma}=\mathbf{0}$ (Proposition). Note that unlike classical linear elasticity, the elastic rotation field can be spatially inhomogeneous even though the stress field vanishes.

In the sequel, plane strain problems (i.e. edge dislocations distributions) are considered, in the plane normal to $\mathbf{x}_{3}$-axis. The non-zero components of the skewsymmetric elastic distorsion are thus $U_{12}^{e}\left(x_{1}, x_{2}\right)=-U_{21}^{e}\left(x_{1}, x_{2}\right)$ and, according to (29), the non-zero component of the vector $\boldsymbol{\varphi}$ is $\varphi_{3}\left(x_{1}, x_{2}\right)$. From (31), the components of the Nye tensor read

$$
\alpha_{13}=\varphi_{3,1} \quad \text { and } \quad \alpha_{23}=\varphi_{3,2} .
$$

It can thus be noted that a ZSE dislocation tensor field must fulfill the condition

$$
\alpha_{13,2}-\alpha_{23,1}=0
$$

\subsubsection{Infinite straight dislocation walls}

Infinite parallel walls of edge dislocations (i.e. tilt walls) is a customary example of ZSE configuration which has been investigated in detail [25]. In the present work, two cases are considered in the context of homogeneous elasticity: a unit-cell containing infinite (i) positive and negative tilt walls (Figure 4a) and (ii) positive tilt walls (Figure 5a). These two distributions of edge dislocations have been modelled as follows

$\alpha_{13}\left(x_{1}\right)=\frac{\partial \varphi_{3}}{\partial x_{1}}\left(x_{1}\right) \quad$ with $\quad \varphi_{3}\left(x_{1}\right)=\frac{k}{4}\left(1+\tanh \left(\frac{x_{1}+a}{\rho}\right)\right)\left(1 \mp \tanh \left(\frac{x_{1}-a}{\rho}\right)\right)$

The distance between the walls is $2 a$ while $\rho$ and $k$ are parameters controlling respectively the spread and the intensity of the walls. FFT computations have been performed on a unit-cell of $512 \times 512$ pixels with $a=128$ pixels, $\rho=2$ and $k=8.10^{7} \mathrm{~m}^{-1}$. For both cases, the obtained numerical stress field is identically zero. The only non-zero component of the $\chi$ field being $\chi_{12}$, the $\nabla z$ field, which solves the local problem (33), does not present diagonal components.

The components of the elastic distorsion field $\mathbf{U}^{e}$ field are reported in Figures $4 \mathrm{~b}-\mathrm{c}$ and 5b-c. The configuration with a null average dislocation density leads to a piecewise constant elastic distorsion field whereas the configuration with a non-vanishing average dislocation density yield a linearly varying elastic distorsion field in the $\mathbf{x}_{1}$ direction. It can be noted that our result for the latter configuration illustrates the fact that the correct stress field is obtained even if the dislocation average over the unit-cell is not zero. However, as discussed in Section 2, the nonperiodic part of the elastic distorsion cannot be retrieved.

Before envisaging other ZSE configurations, it is instructive to consider terminating vertical tilt walls. Indeed, in this case, the partial derivative $\alpha_{13,2}$ is not zero at the ends of the walls. As a consequence, the condition (35) cannot be fulfilled at these points and stresses exist inside the unit-cell (Figure 6). A zerostress field configuration with terminating walls thus necessarily requires $\alpha_{13}$ and $\alpha_{23}$ distributions so that (35) is verified (i.e. vertical and horizontal tilt walls). This is precisely the configuration of dislocation walls introduced by Mura [22]. They are now considered for a further assessment of our FFT numerical framework. 


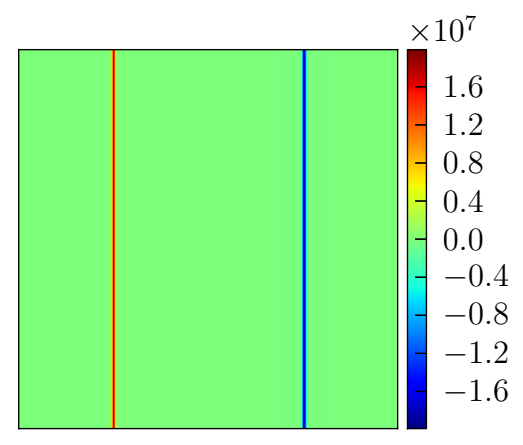

(a) $\alpha_{13}$

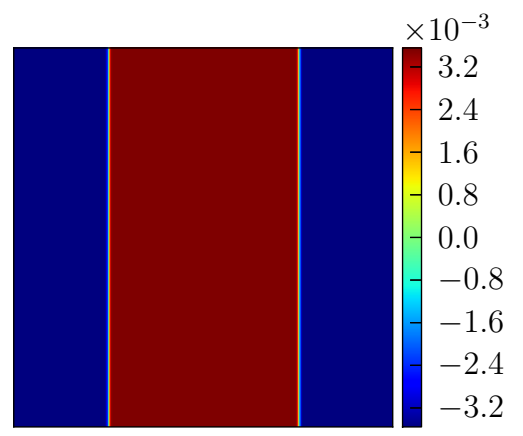

(b) $U_{12}^{e}$

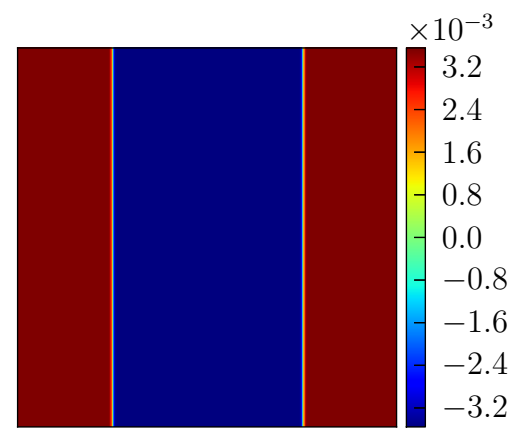

(c) $U_{21}^{e}$

Figure 4. Tilt dislocation walls configuration with $\left\langle\alpha_{13}\right\rangle=0$.

\subsubsection{Cylindrical dislocation walls with rectangular cross-section}

The studied microstructure, composed of finite vertical and horizontal walls, is shown on Figures 7a-b. It has been modeled as follows

$$
\begin{array}{r}
\alpha_{13}\left(x_{1}, x_{2}\right)=\frac{\partial \varphi_{3}}{\partial x_{1}}\left(x_{1}, x_{2}\right) \quad \text { and } \quad \alpha_{23}\left(x_{1}, x_{2}\right)=\frac{\partial \varphi_{3}}{\partial x_{2}}\left(x_{1}, x_{2}\right) \quad \text { with } \\
\varphi_{3}\left(x_{1}, x_{2}\right)=\frac{k}{16}\left(1+\tanh \left(\frac{x_{1}+a}{\rho}\right)\right)\left(1-\tanh \left(\frac{x_{1}-a}{\rho}\right)\right) \\
\left(1+\tanh \left(\frac{x_{2}+b}{\rho}\right)\right)\left(1-\tanh \left(\frac{x_{2}-b}{\rho}\right)\right)
\end{array}
$$

$a$ and $b$ represent the lengths of the horizontal and vertical walls, respectively, and the dislocation microstructure proposed by Mura [22] is obtained in the limit $\rho \rightarrow 0$. In practice, it is necessary to spread the dislocation distribution in order to obtain an accurate numerical estimate of the stress at the corners of the cylindrical wall. The computation parameters are: $a=128$ pixels, $b=64$ pixels, $\rho=2$ and $k=8.10^{7} \mathrm{~m}^{-1}$. As shown in Figure $7 \mathrm{c}$, a precise description of the stress field is obtained with negligible unintended stresses at the corners of the wall. The corresponding elastic distorsion is piecewise constant (Figures 7d-e). As detailed in Appendix D, the $\nabla z$ field counterbalances the symmetric part of the $\chi$ field to keep 


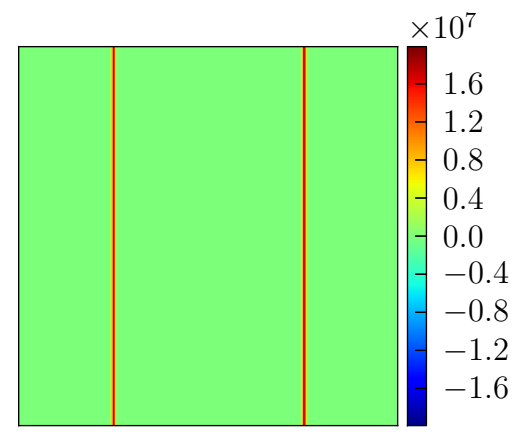

(a) $\alpha_{13}$
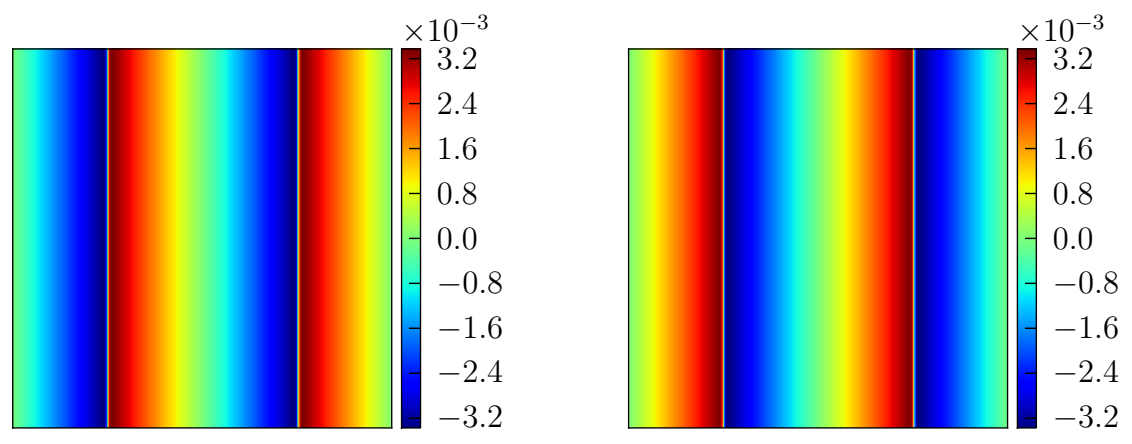

(b) $U_{12}^{e}$

(c) $U_{21}^{e}$

Figure 5. Tilt dislocation walls configuration with $\left\langle\alpha_{13}\right\rangle \neq 0$.

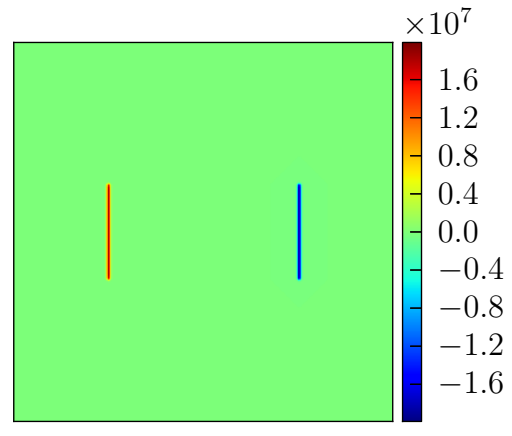

(a) $\alpha_{13}$

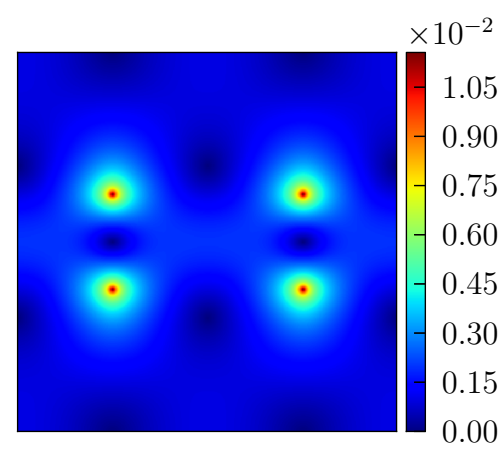

(b) $\sqrt{\sigma_{i j} \sigma_{i j}} / \mu$

Figure 6. Terminating tilt dislocation walls configuration with $\left\langle\alpha_{13}\right\rangle=0$.

the elastic strain to zero and the elastic rotation to the spatially inhomogeneous field (unique up to a uniform skew-symmetric tensor) required by the prescribed dislocation density and the stress-free macroscopic condition. 


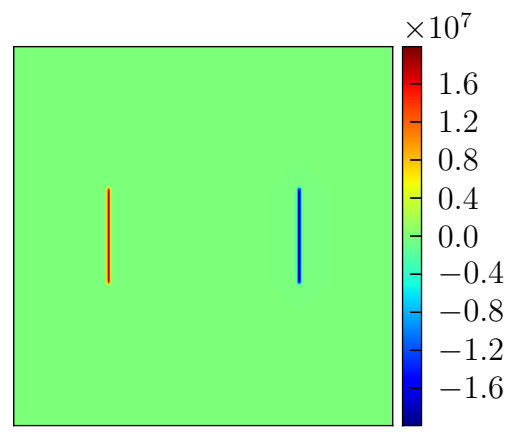

(a) $\alpha_{13}$

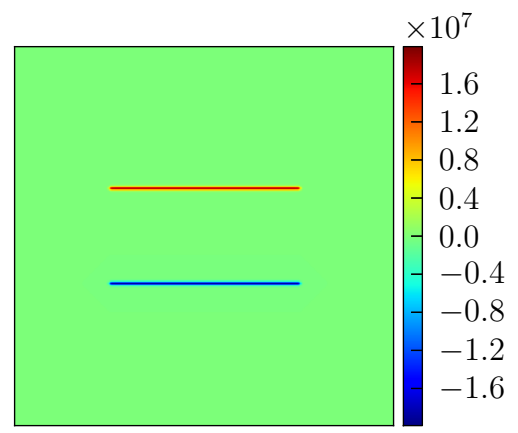

(b) $\alpha_{23}$

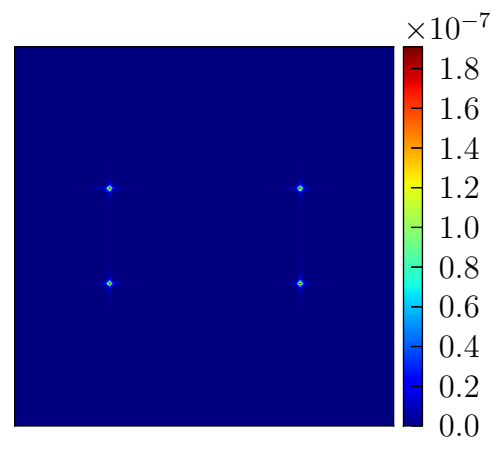

(c) $\sqrt{\sigma_{i j} \sigma_{i j}} / \mu$

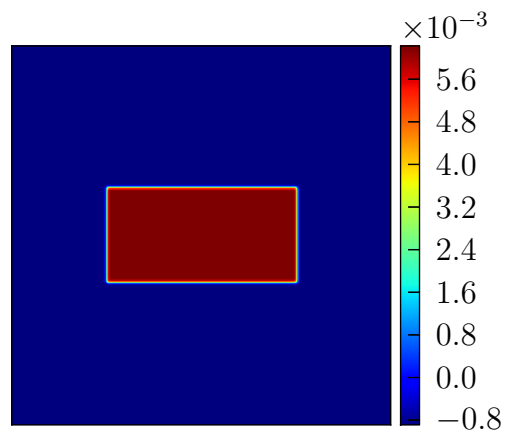

(d) $U_{12}^{e}$

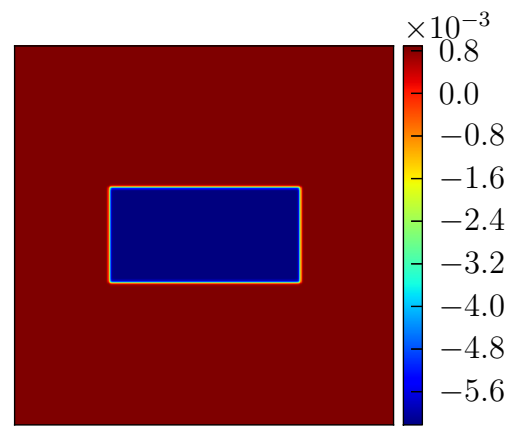

(e) $U_{21}^{e}$

Figure 7. Cylindrical dislocation wall with rectangular cross-section.

\subsubsection{Cylindrical dislocation walls with elliptic cross-section}

We further consider the case of an elliptic dislocation wall described by

$$
\begin{gathered}
\alpha_{13}\left(x_{1}, x_{2}\right)=\frac{\partial \varphi_{3}}{\partial x_{1}}\left(x_{1}, x_{2}\right) \quad \text { and } \quad \alpha_{23}\left(x_{1}, x_{2}\right)=\frac{\partial \varphi_{3}}{\partial x_{2}}\left(x_{1}, x_{2}\right) \quad \text { with } \\
\varphi_{3}\left(x_{1}, x_{2}\right)=\frac{k}{2}\left(1+\tanh \left(\frac{1}{\rho}\left(\sqrt{\left(\frac{x_{1}}{c_{1}}\right)^{2}+\left(\frac{x_{2}}{c_{2}}\right)^{2}}-a\right)\right)\right) .
\end{gathered}
$$


By contrast with the rectangular cross-section, both $\alpha_{13}$ and $\alpha_{23}$ densities are nonzero at each point of the wall (Figures $8 \mathrm{a}-\mathrm{b}$ ). The parameters for this configuration are: $a=64$ pixels, $c_{1}=2, c_{2}=1, \rho=2$ and $k=8.10^{7} \mathrm{~m}^{-1}$. It must be noted that the average of the absolute value of the dislocation density is the same than for the rectangular configuration. With the chosen parameters, we have, in both cases,

$$
\left\langle\left|\alpha_{13}\right|\right\rangle=7.810^{4} \mathrm{~m}^{-1} \quad \text { and } \quad\left\langle\left|\alpha_{23}\right|\right\rangle=2\left\langle\left|\alpha_{13}\right|\right\rangle \text {. }
$$

Once again, a very good numerical description of the stress-free state is obtained with negligible stresses in the wall interfacial zone (Figure 8c) and the elastic distorsion field is piecewise constant (Figures 8d-e).

Finally, it has to be mentioned that similar computations for 3D microstructures, with stress-free ellipsoidal dislocation walls [22], deliver an accurate description of the fields. They are not reported here for conciseness.

\subsection{Infinite bicrystal}

Let us consider the determination of internal (residual) stresses in an infinite bicrystal composed of plastically deformed crystals which are separated by a planar interface. The plastic deformation, characterized by the plastic distorsion $\mathbf{U}^{p}$, is assumed uniform within each crystal. This problem has been considered in [26] for elastically isotropic crystals and [27] for general elastic anisotropy. Analytical expressions were derived in the case of a planar boundary normal to an axis of the reference frame. As noted in [27], the stress field in the infinite bicrystal coincide with the one in a rank-one laminate material composed of the two crystals with equal volume fraction. In the absence of externally applied stress $(\overline{\boldsymbol{\sigma}}=0)$, the internal stresses are homogeneous per crystal and opposite. The stress jump $[\boldsymbol{\sigma}]$ at the planar interface, with arbitrary normal $\mathbf{n}$, can be expressed as (see proof in Appendix C),

$$
[\boldsymbol{\sigma}]=\boldsymbol{\sigma}^{2}-\boldsymbol{\sigma}^{1}=-\left[\mathbf{U}^{p}\right]: \boldsymbol{\Delta}^{1}:\left(\mathbf{I}+\frac{1}{2} \boldsymbol{\Delta}^{1}:\left(\mathbf{S}^{2}-\mathbf{S}^{1}\right)\right)^{-1}
$$

In the case of two elastically isotropic crystals separated by an interface with normal $\mathbf{n}=\mathbf{x}_{1}$, the non-vanishing components of the stress jump read [27]

$$
\left\{\begin{array}{l}
{\left[\sigma_{22}\right]=\frac{2 E^{1} E^{2}\left(\left(E^{1}+E^{2}\right)\left[U_{22}^{p}\right]+\left(\nu^{2} E^{1}+\nu^{1} E^{2}\right)\left[U_{33}^{p}\right]\right)}{\left(E^{2}\left(1+\nu^{1}\right)+E^{1}\left(1+\nu^{2}\right)\right)\left(E^{2}\left(1-\nu^{1}\right)+E^{1}\left(1-\nu^{2}\right)\right)},} \\
{\left[\sigma_{33}\right]=\frac{2 E^{1} E^{2}\left(\left(E^{1}+E^{2}\right)\left[U_{33}^{p}\right]+\left(\nu^{2} E^{1}+\nu^{1} E^{2}\right)\left[U_{22}^{p}\right]\right)}{\left[E^{2}\left(1+\nu^{1}\right)+E^{1}\left(1+\nu^{2}\right)\right]\left[E^{2}\left(1-\nu^{1}\right)+E^{1}\left(1-\nu^{2}\right)\right]},} \\
{\left[\sigma_{23}\right]=4 \frac{\mu^{1} \mu^{2}}{\mu^{1}+\mu^{2}}\left[U_{23}^{p}\right],}
\end{array}\right.
$$

with $E^{i}$ and $\nu^{i}(i=1,2)$ the Young modulus and Poisson coefficient of each crystal. The discontinuity of the $\mathbf{U}^{p}$ field can be alternatively described, using the FrankBilby relation [28], by a two-dimensional dislocation array with density

$$
\boldsymbol{\alpha}=\left[\mathbf{U}^{p}\right] \times \mathbf{n} .
$$




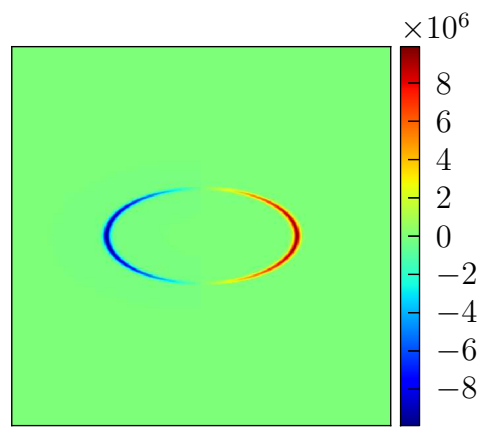

(a) $\alpha_{13}$

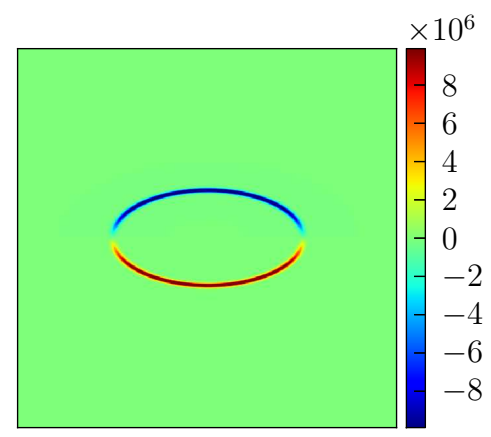

(b) $\alpha_{23}$

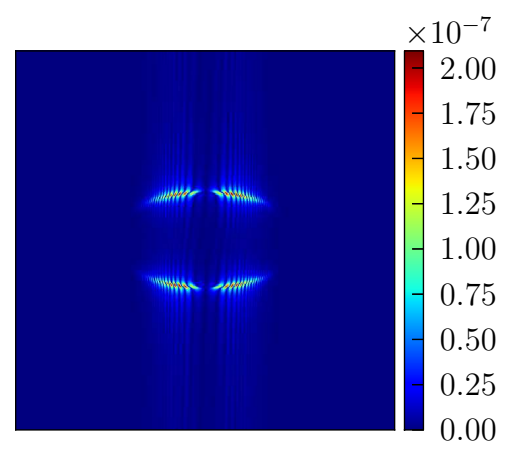

(c) $\sqrt{\sigma_{i j} \sigma_{i j}} / \mu$

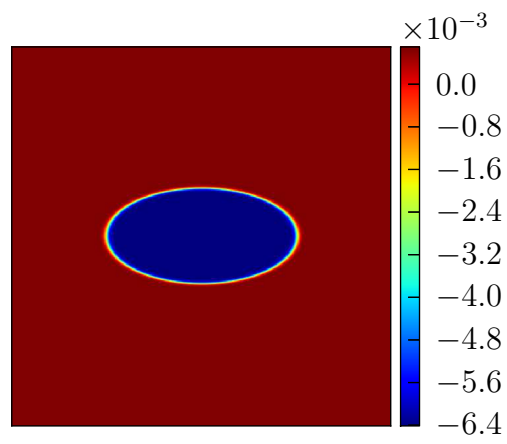

(d) $U_{12}^{e}$

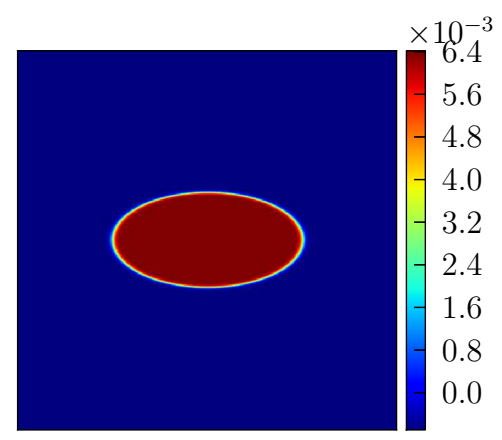

(e) $U_{21}^{e}$

Figure 8. Cylindrical dislocation wall with elliptic cross-section.

The FFT computations have been evaluated by prescribing either a homogeneous plastic distorsion field $\mathbf{U}^{p}$ within each crystal or a dislocation density at the interface by using relation (42). These two calculations are strictly equivalent. It can be noted that in the context of a periodic laminate microstructure, the change of sign of the unit normal to the interface must be taken into account. As a consequence, it is required to consider dislocation arrays with opposite signs in the unit-cell to perform the calculation based on the Frank-Bilby relations. The condition $\langle\boldsymbol{\alpha}\rangle=\mathbf{0}$ is thus fulfilled. An example of unit-cell is given in Figure 9. A very good agreement 
has been obtained between our numerical results and the analytical expressions (41). It has been also verified that the uniformity of the FFT stress field within each crystal is not affected when the normal of the interface is not aligned with an axis of the unit-cell [29].

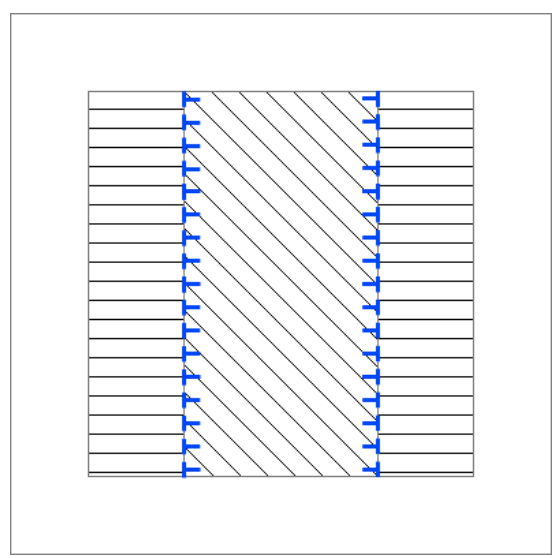

Figure 9. Unit-cell of laminate bicrystal with edge dislocation wall configuration corresponding to homogeneous incompatible distorsion within each crystal with a jump $\left[\chi_{22}\right]$. The interface is normal to $\mathbf{x}_{1}$.

\section{Concluding remarks}

Following Roy and Acharya [4], this work represents a contribution for the numerical implementation of the FDM theory. Our investigation gives an insight into the particular case of periodic media and it has been focused on the accurate determination of the internal stress field for a prescribed dislocation density distribution (i.e. static problem) and macroscopic stress. These results are thus a preliminary step towards a model of plasticity, based on the FDM theory, in the context of periodic microstructures. In this framework, uniqueness properties paralleling the non-periodic case [3] of the stress and elastic distortion fields have been proved and an additional condition (3) on the dislocation tensor (w.r.t. the non-periodic case) has been pointed out. By making use of a Stokes-Helmholtz like decomposition of the elastic distorsion (also used in numerical implementations of FDM in the non-periodic case), it has been shown that the internal stress field can be efficiently evaluated by means of the FFT approach. Problems with stress-free dislocation distributions, which are equilibrium solutions of the FDM theory, are correctly solved and the numerical estimate of the residual stress field in an infinite bicrystal is in agreement with the analytical solution of laminates theory. These outcomes lay the ground for the study of periodic initial-boundary-problems of dislocation-mediated plastic evolution by means of the FFT numerical scheme.

\section{Acknowledgements}

It is a pleasure for AA to acknowledge the hospitality and support of the CNRS, Laboratoire de Mécanique et d'Acoustique, Marseille, during a sabbatical visit in 2008-2009 when this work was initiated. AB gladly acknowledges the support of Université Paris Nord during its scientific stay in Paris in 2012. PS acknowledges the financial support of Labex MEC and of $\mathrm{A}^{*}$ Midex through grants ANR-11LABX-0092 and ANR-11-IDEX-0001-02. 


\section{Appendix A. Useful relations of tensor analysis}

$\boldsymbol{\epsilon}$ is the third-order permutation tensor whose components read

$$
\epsilon_{i j k}=(i-j)(j-k)(k-i) / 2
$$

that is

$$
\epsilon_{i j k}=\left\{\begin{aligned}
+1 & \text { if }(i j k) \text { is an even permutation of }(123) \\
-1 & \text { if }(i j k) \text { is an odd permutation of }(123) \\
0 & \text { otherwise }
\end{aligned}\right.
$$

The cross-product of vectors $\mathbf{v}$ and $\mathbf{w}$ is defined by

$$
\mathbf{v} \times \mathbf{w}=\boldsymbol{\epsilon}:(\mathbf{v} \otimes \mathbf{w}), \quad(\mathbf{v} \times \mathbf{w})_{m}=\epsilon_{m j k} v_{j} w_{k} .
$$

The curl of a vector field $\mathbf{w}$ thus read

$$
\operatorname{curl}(\mathbf{w})=\boldsymbol{\nabla} \times \mathbf{w}=\boldsymbol{\epsilon}:{ }^{\mathrm{T}}(\boldsymbol{\nabla} \mathbf{w}), \quad \operatorname{curl}(\mathbf{w})_{m}=\epsilon_{m j k} w_{k, j} .
$$

By defining $\mathbf{w}=\mathbf{c} . \mathbf{A}, \forall \mathbf{c}$ spatially uniform vector field, we have

$$
\nabla \times(\mathbf{c} . \mathbf{A})=\mathbf{c} .(\nabla \times \mathbf{A})
$$

with the curl of the second-order tensor field $\mathbf{A}$ defined as

$$
\operatorname{curl}(\mathbf{A})=\nabla \times \mathbf{A}, \quad \operatorname{curl}(\mathbf{A})_{i m}=\epsilon_{m j k} A_{i k, j}
$$

The cross-product of the second-order tensor $\mathbf{A}$ and the vector $\mathbf{w}$ is

$$
(\mathbf{A} \times \mathbf{w})_{i m}=\epsilon_{m j k} A_{i j} w_{k}
$$

\section{Appendix B. Elements of analysis for periodic functions [30]}

This appendix summarizes a few mathematical results mostly due to Bourel [30] and useful to prove the Proposition.

Let a unit-cell $V$ allowing to fill the space $\mathbb{R}^{N}$ by translation along $N$ vectors $\mathbf{Y}_{1}, \ldots, \mathbf{Y}_{N}$. $\mathcal{R}$ is the lattice generated by these vectors

$$
\mathcal{R}=\left\{\mathbf{Y}, \mathbf{Y}=\sum_{i=1}^{N} n_{i} \mathbf{Y}_{i}, n_{i} \in \mathbb{Z}\right\}
$$

and $\mathcal{R}_{*}$ is the reciprocal lattice. Define the following spaces of periodic scalar functions:

$$
\begin{aligned}
& L_{\sharp}^{2}(V)=\left\{f \in L_{l o c}^{2}\left(\mathbb{R}^{N}\right), f(\mathbf{x}+\mathbf{Y})=f(\mathbf{x}), \text { a.e. } \mathbf{x} \in V, \quad \forall \mathbf{Y} \in \mathcal{R}\right\}, \\
& H_{\sharp}^{1}(V)=\left\{f \in H_{l o c}^{1}\left(\mathbb{R}^{N}\right), f \in L_{\sharp}^{2}(V), \frac{\partial f}{\partial x_{i}} \in L_{\sharp}^{2}(V) 1 \leq i \leq N,\right\}
\end{aligned}
$$


Similarly, $\boldsymbol{L}_{\sharp}^{2}(V)$ and $\boldsymbol{H}_{\sharp}^{1}(V)$ are composed of vectors fields $\boldsymbol{u}$ with all their components $u_{i}$ in $L_{\sharp}^{2}(V)$ and $H_{\sharp}^{1}(V)$ respectively and, $\mathcal{L}_{\sharp}^{2}(V)$ and $\mathcal{H}_{\sharp}^{1}(V)$ are composed of tensor fields $\chi$ with all their components $\chi_{i j}$ in $L_{\sharp}^{2}(V)$ and $H_{\sharp}^{1}(V)$ respectively. Let $\widehat{f}(\boldsymbol{\xi})$ the Fourier transform of a periodic scalar function $f(\mathbf{x})$. Noting that

$$
\widehat{\nabla f}(\boldsymbol{\xi})=\imath \boldsymbol{\xi} \widehat{f}(\boldsymbol{\xi}),
$$

we have

$$
\begin{cases}\widehat{\nabla \mathbf{u}}(\boldsymbol{\xi})=\imath \boldsymbol{\xi} \otimes \widehat{\mathbf{u}}(\boldsymbol{\xi}), & \widehat{\operatorname{curl}(\mathbf{u})}(\boldsymbol{\xi})=\imath \boldsymbol{\xi} \times \widehat{\mathbf{u}}(\boldsymbol{\xi}), \\ \widehat{\operatorname{div}(\chi)}(\boldsymbol{\xi})=\imath \widehat{\chi}(\boldsymbol{\xi}) \cdot \boldsymbol{\xi}, & \widehat{\operatorname{curl}(\boldsymbol{\chi})}(\boldsymbol{\xi})=\imath \boldsymbol{\xi} \times \widehat{\chi}(\boldsymbol{\xi}) .\end{cases}
$$

Lemma B.1: Let $\mathbf{U} \in \mathcal{L}_{\sharp}^{2}(V)$. Then if $\operatorname{curl}(\mathbf{U})=\mathbf{0}$ there exists $\boldsymbol{z} \in \boldsymbol{H}_{\sharp}^{1}(V)$ such that

$$
\mathbf{U}=\nabla z+\langle\mathbf{U}\rangle
$$

$z$ is unique, up to an additive constant vector.

Proof : Using the Fourier decomposition of $\mathbf{U}$

$$
\mathbf{U}(\mathbf{x})=\sum_{\boldsymbol{\xi} \in \mathcal{R}_{*}} \widehat{\mathbf{U}}(\boldsymbol{\xi}) e^{\imath \boldsymbol{\xi} \cdot \mathbf{x}} \quad \text { with } \sum_{\boldsymbol{\xi} \in \mathcal{R}_{*}}\|\widehat{\mathbf{U}}(\boldsymbol{\xi})\|^{2}<+\infty,
$$

one has, according to (B4):

$$
\boldsymbol{\xi} \times \widehat{\mathbf{U}}(\boldsymbol{\xi})=\mathbf{0},
$$

i.e.

$$
\boldsymbol{\xi} \times \widehat{\mathbf{U}}_{i}^{T}(\boldsymbol{\xi})=\mathbf{0}
$$

where $\widehat{\mathbf{U}}_{i}^{T}(\boldsymbol{\xi})$ is the $i$-th row of $\widehat{\mathbf{U}}(\boldsymbol{\xi})$. Consequently there exists a scalar $a_{i}$ (possibly depending on $\boldsymbol{\xi}$ ) such that

$$
\widehat{\mathbf{U}}_{i}^{T}(\boldsymbol{\xi})=a_{i} \boldsymbol{\xi}, \quad \forall \boldsymbol{\xi} \neq \mathbf{0},
$$

and therefore

$$
\widehat{\mathbf{U}}(\boldsymbol{\xi})=\mathbf{a} \otimes \boldsymbol{\xi}, \quad \forall \boldsymbol{\xi} \neq \mathbf{0} .
$$

In addition

$$
\widehat{\mathbf{U}}(\mathbf{0})=\langle\mathbf{U}\rangle \text {. }
$$

Define the potential field $z$ through its Fourier components:

$$
\widehat{\boldsymbol{z}}(\mathbf{0})=\mathbf{0}, \quad \widehat{\boldsymbol{z}}(\boldsymbol{\xi})=-\imath \frac{\widehat{\mathbf{U}}(\boldsymbol{\xi}) \cdot \boldsymbol{\xi}}{\|\boldsymbol{\xi}\|^{2}}=\mathbf{a} .
$$


Then,

$$
\widehat{\nabla z}(\boldsymbol{\xi})=\imath \widehat{\mathcal{z}}(\boldsymbol{\xi}) \otimes \boldsymbol{\xi}=\mathbf{a} \otimes \boldsymbol{\xi}=\widehat{\mathbf{U}}(\boldsymbol{\xi}), \quad \forall \boldsymbol{\xi} \neq \mathbf{0}
$$

The only difference in the Fourier components of $\boldsymbol{\nabla} z$ and $\mathbf{U}$ is at frequency $\boldsymbol{\xi}=\mathbf{0}$ where

$$
\widehat{\nabla z}(\xi)=\mathbf{0}=\widehat{\mathbf{U}}(\mathbf{0})-\langle\mathbf{U}\rangle
$$

Therefore

$$
\nabla z(\mathbf{x})=\mathbf{U}(\mathbf{x})-\langle\mathbf{U}\rangle
$$

It can easily be seen that:

$$
\sum_{\boldsymbol{\xi} \in \mathcal{R}_{*}}\|\widehat{\boldsymbol{z}}(\boldsymbol{\xi})\|^{2}<+\infty, \quad \sum_{\boldsymbol{\xi} \in \mathcal{R}_{*}}\|\widehat{\boldsymbol{z}}(\boldsymbol{\xi}) \otimes \boldsymbol{\xi}\|^{2}<+\infty
$$

which proves that $z \in \boldsymbol{H}_{\sharp}^{1}(V)$. $V$ being a connected domain, $z$ is defined up to a constant vector (the average of $\boldsymbol{z}$ was arbitrary chosen to be $\mathbf{0}$ by the choice of $\widehat{z}(\mathbf{0}))$.

Lemma B.2: Let $\boldsymbol{\alpha}$ be a second-order tensor field such that

$$
\boldsymbol{\alpha} \in \mathcal{L}_{\sharp}^{2}(V), \quad \operatorname{div}(\boldsymbol{\alpha})=\mathbf{0} \text { in } \mathbb{R}^{N}, \quad\langle\boldsymbol{\alpha}\rangle=\mathbf{0} .
$$

There exists a second-order tensor field $\boldsymbol{\chi} \in \mathcal{H}_{\sharp}^{1}(V)$ such that:

$$
\operatorname{curl}(\chi)=\boldsymbol{\alpha}, \quad \operatorname{div}(\chi)=\mathbf{0} \quad \text { in } V
$$

$\chi$ is unique up to a constant second-order tensor.

Proof: One has, according to the divergence-free condition expressed in Fourier space

$$
\widehat{\boldsymbol{\alpha}}(\boldsymbol{\xi}) \cdot \boldsymbol{\xi}=\mathbf{0}
$$

Set

$$
\widehat{\chi}(\mathbf{0})=\mathbf{0}, \quad \widehat{\chi}(\boldsymbol{\xi})=-\imath \frac{\widehat{\boldsymbol{\alpha}}(\boldsymbol{\xi}) \times \boldsymbol{\xi}}{\|\boldsymbol{\xi}\|^{2}}
$$

With the above definition, one has

$$
\widehat{\chi}(\boldsymbol{\xi}) \cdot \boldsymbol{\xi}=\mathbf{0}, \quad \forall \boldsymbol{\xi}
$$

Therefore

$$
\operatorname{div}(\chi)=\mathbf{0} \quad \text { in } \mathbb{R}^{N} .
$$


Moreover, according to (B4):

$$
\widehat{\operatorname{curl}(\chi)})(\boldsymbol{\xi})=\imath \boldsymbol{\xi} \times \frac{(\widehat{\boldsymbol{\alpha}}(\boldsymbol{\xi}) \times \boldsymbol{\xi})}{\imath\|\boldsymbol{\xi}\|^{2}}=\widehat{\boldsymbol{\alpha}}(\boldsymbol{\xi})-\frac{(\widehat{\boldsymbol{\alpha}}(\boldsymbol{\xi}) \cdot \boldsymbol{\xi})}{\|\boldsymbol{\xi}\|^{2}} \otimes \boldsymbol{\xi}=\widehat{\boldsymbol{\alpha}}(\boldsymbol{\xi}), \quad \forall \boldsymbol{\xi} \neq \mathbf{0}
$$

All Fourier components of $\operatorname{curl}(\chi)$ and $\boldsymbol{\alpha}$ coincide at frequency $\boldsymbol{\xi} \neq \mathbf{0}$. When $\boldsymbol{\xi}=\mathbf{0}$, the periodicity of $\chi$ implies that $\widehat{\operatorname{curl}(\boldsymbol{\chi}})(\mathbf{0})=\mathbf{0}$ and since $\widehat{\boldsymbol{\alpha}}(\mathbf{0})=\mathbf{0}$ all Fourier components of $\operatorname{curl}(\chi)$ and $\boldsymbol{\alpha}$ coincide.

Regarding the uniqueness of $\chi$, if two fields $\chi_{1}$ and $\chi_{2}$ are solutions of (B7), their difference $\boldsymbol{\delta} \boldsymbol{\chi}$ is curl-free and according to lemma B.1 can be written as

$$
\delta \chi=\nabla z+\langle\delta \chi\rangle
$$

where $\boldsymbol{z}$ is periodic. But $\boldsymbol{\delta} \boldsymbol{\chi}$ is also divergence-free and therefore the periodic field $z$ satisfies

$$
\Delta z=\mathbf{0}
$$

Therefore $\boldsymbol{z}$ is a constant vector (standard result). Therefore $\boldsymbol{\nabla} z=\mathbf{0}$ and $\boldsymbol{\delta} \boldsymbol{\chi}$ is a constant second-order tensor.

\section{Appendix C. Residual stresses in two-phase rank-one laminate materials}

Let us consider a two-phase composite with "thermoelastic" constituents (i.e. elastic behaviour with eigenstrains). The volume fraction of phase $r$ is $f_{r}$. The local and overall consitutive relations respectively read

$$
\varepsilon(\mathbf{x})=\mathbf{S}(\mathbf{x}): \boldsymbol{\sigma}(\mathbf{x})+\varepsilon_{*}(\mathbf{x}) \text { and } \bar{\varepsilon}=\widetilde{\mathbf{S}}: \overline{\boldsymbol{\sigma}}+\widetilde{\boldsymbol{\varepsilon}}_{*}
$$

with $\mathbf{S}(\mathbf{x})=\sum_{r=1}^{2} \chi^{r}(\mathbf{x}) \mathbf{S}^{r}$ and $\boldsymbol{\varepsilon}_{*}(\mathbf{x})=\sum_{r=1}^{2} \chi^{r}(\mathbf{x}) \boldsymbol{\varepsilon}_{*}{ }^{r} \cdot \chi^{r}(\mathbf{x})$ is the characteristic function of the phase $r$ while $\mathbf{S}^{r}$ and $\varepsilon_{*}^{r}$ represent its uniform properties (elastic compliance and eigenstrain tensors). Classically, the overall compliance and eigenstrain tensors are given by

$$
\left\{\begin{array}{l}
\widetilde{\mathbf{S}}=\langle\mathbf{S}: \mathbf{B}\rangle=\sum_{r=1}^{2} f_{r} \mathbf{S}^{r}:\langle\mathbf{B}\rangle^{r} \\
\widetilde{\boldsymbol{\varepsilon}}_{*}=\left\langle\varepsilon_{*}: \mathbf{B}\right\rangle=\sum_{r=1}^{2} f_{r} \varepsilon_{*}^{r}:\langle\mathbf{B}\rangle^{r}
\end{array}\right.
$$

with $\mathbf{B}(\mathbf{x})$ the stress influence tensors relating the local stress $\boldsymbol{\sigma}(\mathbf{x})$ to the macroscopic stress $\overline{\boldsymbol{\sigma}}$. When no macroscopic stress is applied $\left(\overline{\boldsymbol{\sigma}}=0, \overline{\boldsymbol{\varepsilon}}=\widetilde{\boldsymbol{\varepsilon}}_{*}\right)$, the average residual stresses in each phase, resulting from the incompatibility of the eigenstrains, may be written as

$$
\langle\boldsymbol{\sigma}\rangle^{1}=\frac{1}{f_{1}}\left(\mathbf{S}^{1}-\mathbf{S}^{2}\right)^{-1}:\left(\widetilde{\varepsilon}_{*}-\left\langle\varepsilon_{*}\right\rangle\right),\langle\boldsymbol{\sigma}\rangle^{2}=\frac{1}{f_{2}}\left(\mathbf{S}^{2}-\mathbf{S}^{1}\right)^{-1}:\left(\widetilde{\varepsilon}_{*}-\left\langle\varepsilon_{*}\right\rangle\right) .
$$

In the specific case of laminate materials, the stress influence tensors $\mathbf{B}$ are uniform within each phase. By applying the Hill-Laws theorem [31, 32], the stress discontinuity at an interface with unit normal $\mathbf{n}$, for the purely elastic problem (i.e. 
$\overline{\boldsymbol{\sigma}} \neq \mathbf{0}$ and $\left.\boldsymbol{\varepsilon}_{*}=\mathbf{0}\right)$, can be expressed as

$$
[\boldsymbol{\sigma}]=\boldsymbol{\sigma}^{2}-\boldsymbol{\sigma}^{1}=-\boldsymbol{\Delta}^{1}(\mathbf{n}):\left(\mathbf{S}^{2}-\mathbf{S}^{1}\right): \boldsymbol{\sigma}^{2}
$$

where $\boldsymbol{\Delta}^{1}(\mathbf{n})$ is a stress interfacial operator defined by

$$
\boldsymbol{\Delta}^{1}(\mathbf{n})=\mathbf{C}^{1}:\left(\mathbf{I}-\boldsymbol{\Gamma}^{1}(\mathbf{n}): \mathbf{C}^{1}\right) \quad \text { with } \quad \boldsymbol{\Gamma}^{1}(\mathbf{n})=\left[\mathbf{n} \otimes\left(\mathbf{n} \cdot \mathbf{C}^{1} \cdot \mathbf{n}\right)^{-1} \otimes \mathbf{n}\right]^{(s)}
$$

$\mathbf{C}^{1}$ is the elastic moduli tensor of phase 1 and the notation [.] ${ }^{(s)}$ indicates a double (minor and major) symmetrization. From (C4), it follows that

$$
\mathbf{B}^{2}=\left(\mathbf{I}+f_{1} \boldsymbol{\Delta}^{1}:\left(\mathbf{S}^{2}-\mathbf{S}^{1}\right)\right)^{-1}, \quad \mathbf{B}^{1}=\frac{1}{f_{1}}\left(\mathbf{I}-f_{2} \mathbf{B}^{2}\right) .
$$

Finally, by introducing the eigenstrain discontinuity $\left[\varepsilon_{*}\right]$ at the interface, we have

$$
\widetilde{\varepsilon}_{*}-\left\langle\varepsilon_{*}\right\rangle=f_{2}\left[\varepsilon_{*}\right]:\left(\mathbf{B}^{2}-\mathbf{I}\right)
$$

which leads to the result (40).

\section{Appendix D. Incompatible and compatible parts of the elastic distorsion field for cylindrical dislocation walls}

The components of the $\chi$ and $\boldsymbol{\nabla} z$ fields, which give the elastic distorsion, are shown on Figures (D1) and (D2) for the rectangular and elliptic cylindrical walls, respectively.

\section{References}

[1] A. Acharya, J. Mech. Phys. Solids 49 (2001), pp. 761-784.

[2] A. Acharya, J. Mech. Phys. Solids 52 (2004), pp. 301-316.

[3] J.R. Willis, Int. J. Engng Sci. 5 (1967), pp. 171-190.

[4] A. Roy and A. Acharya, J. Mech. Phys. Solids 53 (2005), pp. 143-170.

[5] A. Roy and A. Acharya, J. Mech. Phys. Solids 54 (2006), pp. 1711-1743.

[6] H. Moulinec and P. Suquet, Comput. Methods Appl. Mech. Engrg. 157 (1998), pp. 69-94.

[7] R.A. Lebensohn, Acta Mater. 49 (2001), pp. 2723-2737.

[8] R. Brenner, R.A. Lebensohn, and O. Castelnau, Int. J. Solids Struct. 46 (2009), pp. 30183026.

[9] A. Belkhabbaz, R. Brenner, N. Rupin, B. Bacroix, and J. Fonseca, Procedia Engineering 10 (2011), pp. 1883-1888.

[10] P. Suquet, H. Moulinec, O. Castelnau, M. Montagnat, N. Lahellec, F. Grennerat, P. Duval, and R. Brenner, Procedia IUTAM 3 (2012), pp. 64-78.

[11] J.F. Nye, Acta Metall. 1 (1953), pp. 153-162.

[12] R. Temam, Mathematical problems in Plasticity, Gauthier-Villars, Paris, 1985.

[13] G.W. Milton, The theory of composites, Cambridge University Press, 2002.

[14] H. Moulinec and F. Silva, Int. J. Num. Meth. Engng (2013), submitted.

[15] J. P. Hirth and J. Lothe, Theory of dislocations, Wiley, 1982.

[16] C. E. Inglis, Trans. Instn Naval Archit. 55 (1913), pp. 219-230.

[17] A. A. Griffith, Phil. Trans. R. Soc. Lond. A. 221 (1921), pp. 163-198.

[18] J. Weertman, Dislocation based fracture mechanics, World Scientific Publishing, 1996.

[19] D. A. Hills, P. A. Kelly, D. N. Dai, and A. M. Korsunsky, Solution of crack problems. The distributed dislocation technique, Kluwer Academic Publishers, 1996.

[20] C. Fressengeas and V. Taupin (2013), submitted.

[21] I. N. Sneddon, Proc. R. Soc. Lond. A187 (1946), pp. 229-260. 
[22] T. Mura, Mater. Sci. Eng. A 113 (1989), pp. 149-152.

[23] A. K. Head, S.D. Howison, J. R. Ockendon, and S. P. Tighe, SIAM review 35 (1993), pp. 580-609.

[24] S. Limkumnerd and J. P. Sethna, Phys. Rev. B 75 (2007), p. 224121.

[25] J.P. Hirth, D. M. Barnett, and J. Lothe, Phil. Mag. A 40 (1979), pp. 39-47.

[26] C. Rey and A. Zaoui, Acta Metall. 28 (1980), pp. 687-697.

[27] J. Gemperlova, V. Paidar, and F. Kroupa, Czech. J. Phys. B 39 (1989), pp. 427-446.

[28] A. P. Sutton and R. W. Balluffi, Interfaces in crystalline materials, Oxford University Press, 1995.

[29] P. Suquet and H. Moulinec, Contraintes aux joints de grains dans un bicristal périodique, 2013. Pers. Comm.

[30] C. Bourel, Étude mathématique et numérique de cristaux photoniques fortement contrastés, Ph.D. thesis, Université de Toulon et du Var, France, 2010.

[31] R. Hill, An invariant treatment of interfacial discontinuities in elastic composites, in Continuum mechanics and related problems of analysis, Muskhelishvili 80th Anniversary, 1972, pp. 597-604.

[32] N. Laws, J. Elasticity 5 (1975), pp. 227-235. 


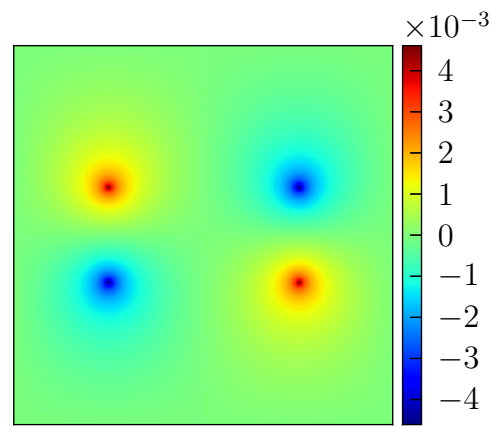

(a) $\chi_{11}$

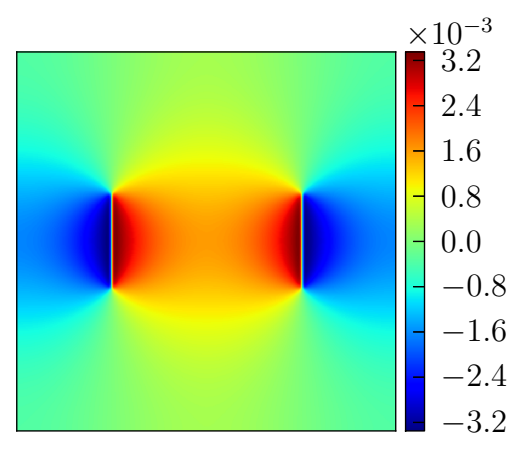

(c) $\chi_{12}$

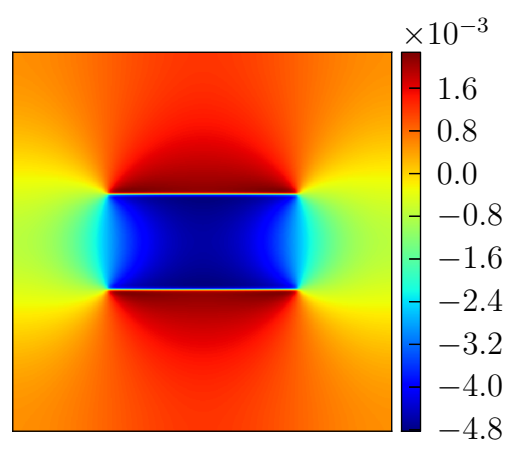

(e) $\chi_{21}$

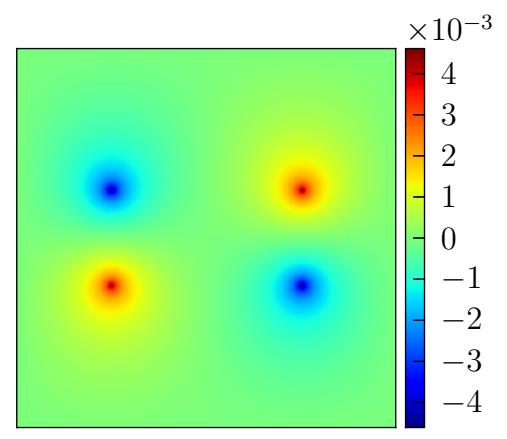

(g) $\chi_{22}$

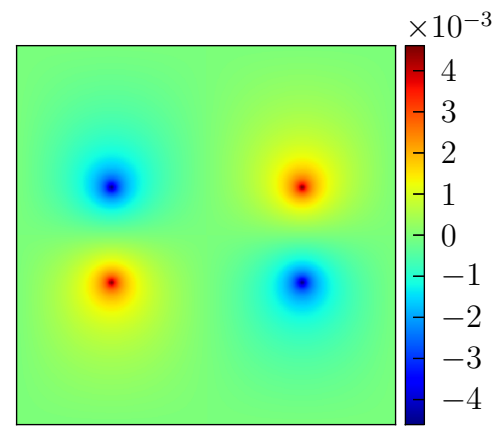

(b) $\nabla z_{11}$

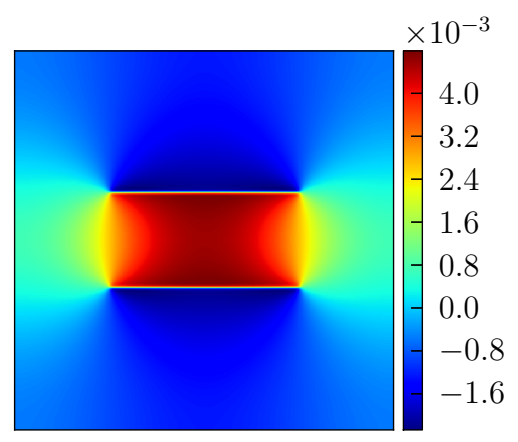

(d) $\nabla z_{12}$

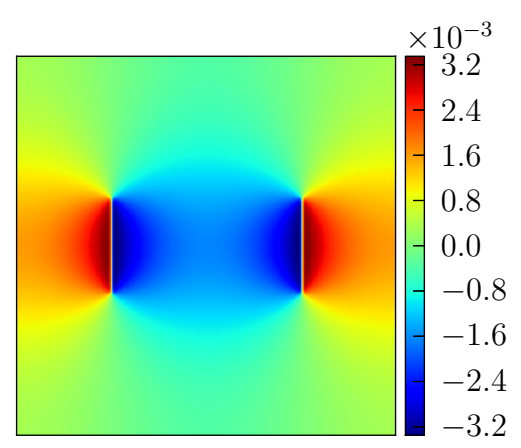

(f) $\nabla z_{21}$

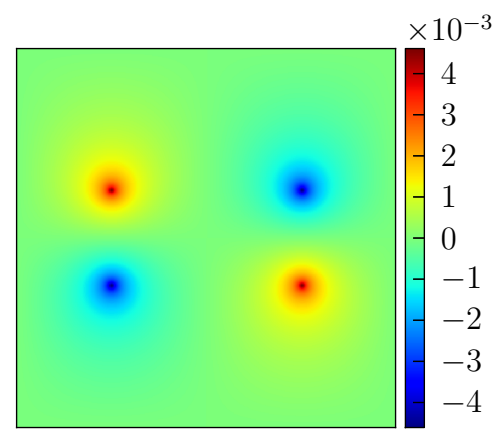

(h) $\nabla z_{22}$

Figure D1. Decomposition of the elastic distorsion for a cylindrical dislocation wall with rectangular cross-section. 


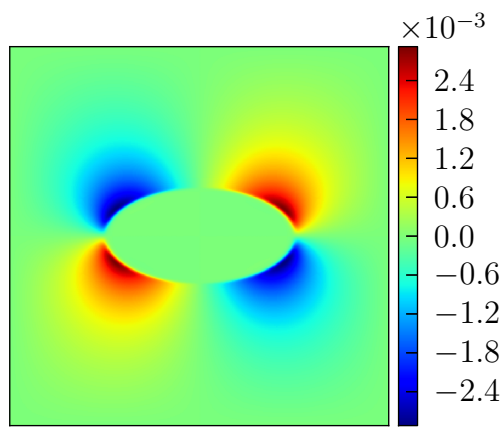

(a) $\chi_{11}$

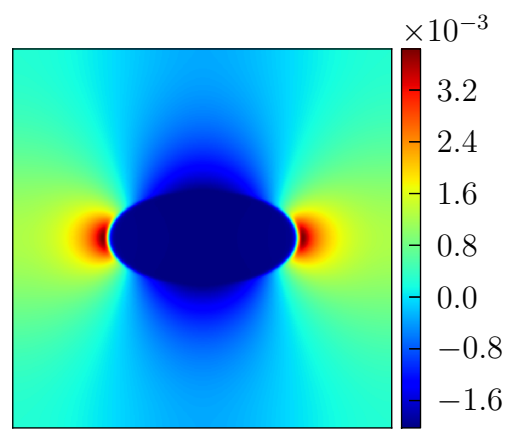

(c) $\chi_{12}$

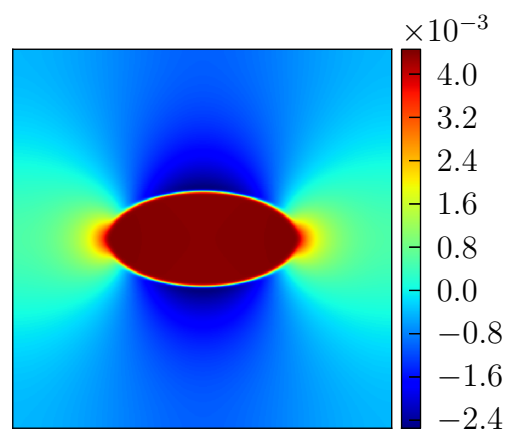

(e) $\chi_{21}$

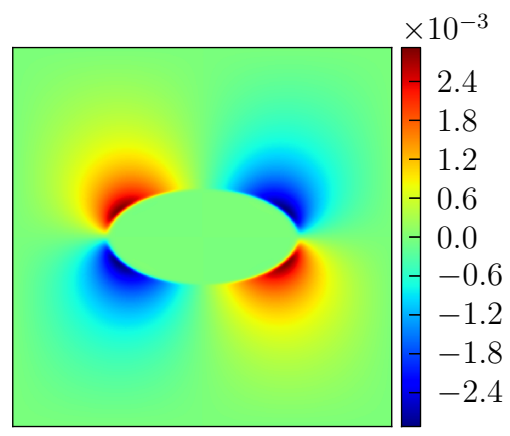

(g) $\chi_{22}$

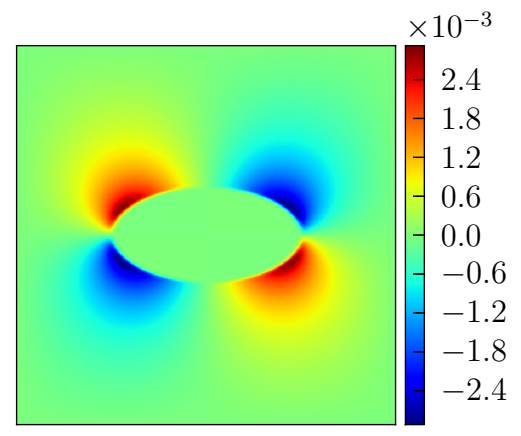

(b) $\nabla z_{11}$

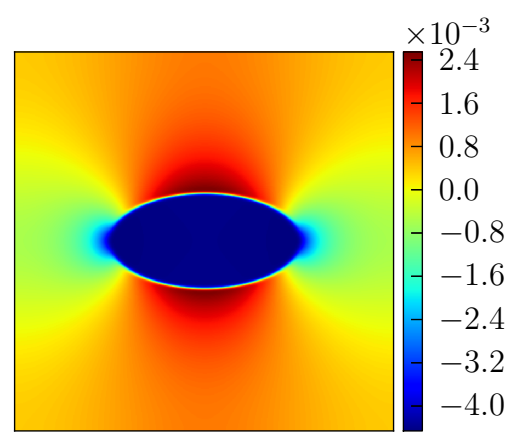

(d) $\nabla z_{12}$

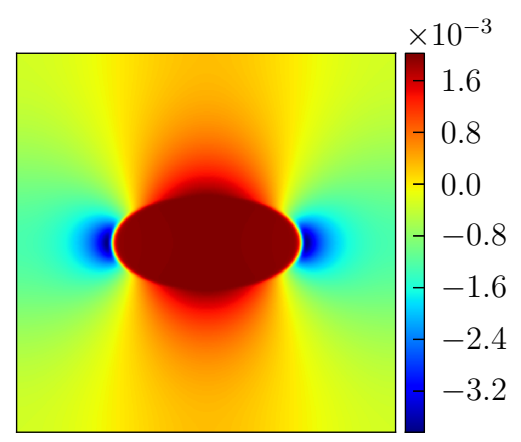

(f) $\nabla z_{21}$

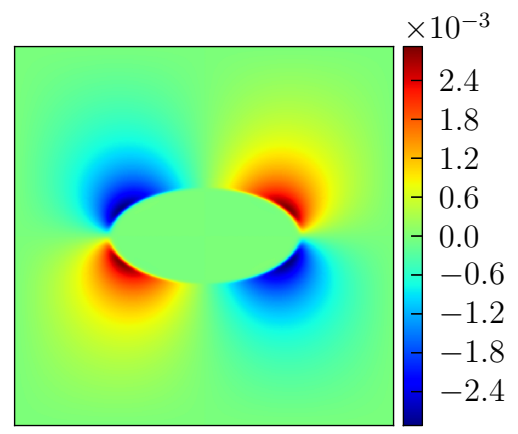

(h) $\nabla z_{22}$

Figure D2. Decomposition of the elastic distorsion for a cylindrical dislocation wall with elliptic crosssection. 